\title{
The Vibration of Two-Dimensional Imperfect Functionally Graded (2D-FG) Porous Rotating Nanobeams based on General Nonlocal Theory A. Rahmani ${ }^{1}$, S. Faroughi ${ }^{*{ }^{* 1}}$, M.I. Friswell ${ }^{2}$ \\ ${ }^{1}$ Faculty of Mechanical Engineering, Urmia University of Technology, Urmia, Iran \\ ${ }^{2}$ College of Engineering, Swansea University, Swansea, UK
}

\begin{abstract}
A comprehensive vibrational analysis of bi-directional functionally graded (2D-FG) rotating nanobeams with porosities is studied for the first time. The beam is modeled based on general nonlocal theory (GNT) where the beam governing equations are derived depending on two different nonlocal parameters. Unlike Eringen's conventional form of nonlocal theory, the general nonlocal theory can reveal both hardening and softening behaviors of the material. Here, the attenuation functions are altered in both transverse and longitudinal directions of 2D-FG nanobeam. This feature, which has a significant effect on the vibrational characteristics, has not been considered in previous studies. Moreover, to estimate the effects of the higher-order transverse shear strains on the vibration of the nanobeam, Reddy's beam theory (RBT), which includes higher-order shear deformation, is employed. The material properties of the 2D-FG rotating nanobeam vary both in the length and thickness directions according to a power law. The generalized differential quadrature method (GDQM) is used to predict the vibration response. Also, the effects of material variation along the length and thickness directions, the rotating velocity of the nanobeam, the porosity volume fraction and the length to thickness ratio of the rotating nanobeam are illustrated and discussed in detail. The investigations performed in this study expose new phenomena for the vibration of nanobeams.
\end{abstract}

\footnotetext{
${ }^{1}$ Corresponding author: Shirko Faroughi (sh.farughi@uut.ac.ir)
} 
Keywords: Two-Dimensional Functionally Graded Nanobeams; Rotating Nanobeams; General Nonlocal Elasticity; Reddy's Beam Theory; GDQM

\section{Introduction}

Due to the outstanding mechanical, thermal and electrical properties of micro/nano structures, their applications in small-scale systems such as microsensors, micro-actuators and microresonators have increased progressively over the past decade. In order to properly design these devices and to increase their reliability, the accurate evaluation of the mechanical behavior of nanostructures, such as bending, buckling, vibration and wave propagation, is essential. Due to the size dependency of the mechanical properties of nanostructures, the classical theories of continuum mechanics are incapable of predicting their mechanical behaviors. Therefore, scientists have proposed modified elasticity theories to resolve the problem and to extract the most accurate results, for example strain gradient elasticity, couple stress, Eringen's nonlocal elasticity and general nonlocal elasticity [1-4]. In recent years, the static and dynamic analysis of micro/nano structures has been extensively investigated by many researchers [5-9]. Since beams are key elements of most micro/nano structures, the following literature review concentrates on investigations into the vibrational behavior of micro/nano beams based on different theories.

Roque et al. [10] employed Eringen's nonlocal theory (ENT) to determine the bending, buckling and vibrational behavior of nanobeams via Timoshenko beam theory (TBT), using an effective meshless method. Ansari et al. [11] studied free vibration of a functional graded material (FGM) Timoshenko microbeam based on strain gradient theory (SGT). They compared the results obtained via classical theory (CT), modified couple stress theory (MCST), and SGT. Thai et al. [12] presented the analysis of the bending, buckling, and vibration of a nanobeam based on Eringen's theory and a shear deformation beam model. Their model is very similar to the nonlocal Euler-Bernoulli beam model and does not require a shear correction factor. ArandaRuiz et al. [13] presented the free vibration of a rotating non-uniform nano-cantilever by applying ENT. They considered the effects of small-scale nonlocal phenomena and angular speed on the vibrational behavior of nanobeams. Sourki and Hosseini [14] considered the vibration analysis of a weakened Euler-Bernoulli nanobeam containing surface effects. They included nonlocal effects and employed MSCT for their model. Babaei and Rahmani [15] 
investigated the lateral vibration of a microbeam under thermal stresses based on MCST and TBT. Khaniki [16, 17] applied mixed local/nonlocal Eringen elasticity to study the transverse vibration behavior of a rotating Euler-Bernoulli cantilever beam.

Some papers in the literature have focused on rotating functionally graded micro/nano beams. FGMs are typically composed of metal and ceramic and continuously alter their material composition from one surface to another to attain desirable characteristics. In recent decades, the applications of FGMs have increased dramatically and the mechanical behavior of FGM micro/nano structures, such as bending, buckling, vibration, and wave propagation, have been investigated by many scientists. Ebrahimi and Barati considered the vibration of FG nanobeams [18] and flexoelectric nanobeams [19]. Roque et al. [20] presented the influence of small-scale parameters on the vibrational behavior of Timoshenko FGM nanobeams. Shafiei and Lin [21] studied the vibrational behavior of bi-directional FG nano-tubes with thermal effects. They applied a nonlocal strain gradient approach based on higher order theory with GDQM to extract the model and the results. Pradhan and Murmu [22] studied the flap-wise bending and vibration behavior of a rotating clamped-free nanobeam based on Eringen's model. Vibrational studies of rotating nanobeams, including the effects of an elastic foundation and the thermal environment was undertaken by Mohammadi et al. [23]. Ebrahimi and Shafei [24] modeled the vibrational behavior of rotating FG nano-beams via ENT. Shafiei et al. [25] investigated the vibrational behavior of bi-dimensional FG nanobeams by applying both couple stress and Eringen's theory separately. They considered two different kinds of porous materials with various boundary conditions. Recently, Tang et al. [26-29] investigated the mechanical behavior of 2D-FG beams and nano-beams, such as nonlinear vibration, post buckling and nonlinear bending. Mirjavadi et al. [30] studied the effects of porosity and temperature on the vibrational behavior of bidirectional FG microbeams; they used MCST and TBT. Ebrahimi and Barati [31] studied the vibrational behavior of higher-order FG nanobeams using a nonlocal MCST. Shafiei et al. [32] proposed flap-wise bending vibration analysis of a rotary tapered FG nanobeam in a thermal environment. They employed ENT and EBT models, utilized the differential quadrature method (DQM), and carefully imposed the cantilever boundary conditions.

Fang et al. [33, 34] developed a three-dimensional model of rotating FG small scale beams, using MCST and Euler-Bernoulli beam models. They utilized Lagrange's equations and the Ritz 
method to model and analyze the axial, chord-wise, and flap-wise motions. Talebitooti et al. [35] considered the semi-analytical vibration analysis of a rotating tapered axially FG nanobeam. They applied ENT and the differential transformation method to extract the numerical results. Bhattacharya and Das [36] investigated the free vibrational behavior of a bi-directional FG double-tapered rotating micro-beam. They developed the model on the basis of TBT and MSCT. Aria and Friswell [37] investigated a nonlocal FE model for buckling and free vibrational behaviors of FG nanobeams based on a first-order shear deformation beam model. Khaniki [38] employed a modified two-phase Eringen's model to determine the vibrational behavior of axially FG Euler-Bernoulli nanobeams. Atanasov and Stojanović [39] studied forced vibration analysis of a rotating nonlocal cantilever nano-beam. They utilized ENT and EBT to establish the model and applied the Galerkin method and standard modal analysis to extract the results.

Based on the literature, the applications of rotating micro/nanostructures including micro/nano multiple gear systems, micro/nano gears, micro/nano turbines, micro/nano blades, micro/nano rotating rings and micro/nano robots have been developed extensively. For instance, cantilever nano beams have been employed in atomic microscopes and accelerometers, and clampedclamped beams are utilized in micro-mirror systems. Kiani and Soltani [40] investigated the longitudinal, flap-wise, and chord-wise vibrational behavior of rotary doubly coaxial/non-coaxial nano beams as nano motors. They developed the governing equations using nonlocal Rayleigh and Timoshenko beams. Yang et al. [41] presented the nonlinear bending, buckling and vibration of nano-beams. They employed a nonlocal Euler-Bernoulli beam model and von Karman geometric nonlinearity to establish the governing equation of motion, and utilized DQM to extract the results.

Recently, Shaat $[4,42]$ confirmed that ENT has some serious limitations for materials with different nonlocal transverse and longitudinal behavior; hence they proposed the general nonlocal theory (GNT). The GNT is established on the basis of the difference between the shear and normal strains in the nonlocal fields. The transverse and the longitudinal acoustic dispersions of materials can be considered simultaneously in GNT. Also, GNT can model the effects of Poisson's ratio on the mechanical behavior of different materials $[43,44]$.

The literature demonstrates that the existing investigations on the vibrational behavior of FG small-scale beams are based on ENT, SGT and MCST. But GNT has not yet been considered for 
the analysis of the vibrational behavior of micro/nano-beams in any contribution. Furthermore, a comprehensive study including the higher-order-shear deformation beam model, 2D FGM materials, a rotating nano-beam with CC boundary conditions, and general nonlocal elasticity has not been developed.

In this study, a comprehensive vibrational analysis of 2D-FG rotating nanobeams with porosities is developed based on GNT and RBT for the first time. For this purpose, a novel hybrid model considering the long-range interatomic, as well as the 2D-FG porous model, is developed. The interatomic reaction problem is included in the modeling of size-dependent structures via GNT. The 2D-FG porous material model is considered due to the porosity of the material, which includes the effects via power indices of the FG along both the thickness $(n z)$ and length $(n x)$ directions. GDQM is used to obtain the numerical solutions; GDQM is preferred over finite element analysis due to its faster convergence and reduced computational requirements. Moreover, a parametric investigation is used to indicate the effects of different nonlocal parameters, and the 2D-FG power indices ( $n x$ and $n z$ ), on the vibration of $2 \mathrm{D}$ porous rotating nanobeams.

\section{2- Governing Equations of Motion}

\subsection{D-FG Material}

As shown in Fig. 1, the nano blades are considered to be 2D-FG rotating nanobeams and composed of ceramic and metal whose volume fraction changes in both the $z$ and $x$ directions; therefore, the material configuration alters along both the axial and thickness directions. Hence, the mechanical properties of the nanobeams, such as Lame's constants, alter along the longitudinal ( $x$-axis) and thickness directions ( $z$-axis). 


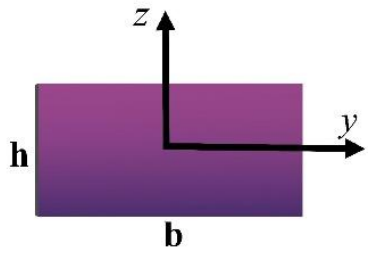

Fig 1. Schematic of the 2D-FG nanobeam rotating with angular velocity $\widetilde{\omega}$

The porous 2D-FG nanobeam is assumed to have either even or uneven porosity distributions across thickness of the beam. For a 2D-FG nanobeam with an even diffusion of porosities, Lame's constants and mass density are expressed as:

$$
\begin{aligned}
& \lambda(x, z)=\lambda_{m}+\left(\lambda_{c}-\lambda_{m}\right)\left(\frac{1}{2}+\frac{z}{h}\right)^{n z}\left(\frac{x}{L}\right)^{n x}-\frac{\gamma}{2}\left(\lambda_{c}+\lambda_{m}\right) \\
& \mu(x, z)=\mu_{m}+\left(\mu_{c}-\mu_{m}\right)\left(\frac{1}{2}+\frac{z}{h}\right)^{n z}\left(\frac{x}{L}\right)^{n x}-\frac{\gamma}{2}\left(\mu_{c}+\mu_{m}\right) \\
& \rho(x, z)=\rho_{m}+\left(\rho_{c}-\rho_{m}\right)\left(\frac{1}{2}+\frac{z}{h}\right)^{n z}\left(\frac{x}{L}\right)^{n x}-\frac{\gamma}{2}\left(\rho_{c}+\rho_{m}\right)
\end{aligned}
$$

The ()$_{m}$ and ()$_{c}$ subscripts are the metal and ceramic properties respectively, $n x$ and $n z$ denote the power law indices and $\gamma$ is the porosity volume fraction. If $\gamma=0, n z=0$ and $n x=$ 0 , the nano beam is constructed from pure ceramic.

The displacement field at point $(x, z)$ of the nanobeam is stated based on RBT as:

$$
\begin{aligned}
& u_{x}(x, z, t)=u^{0}(x)+z \psi(x)-\frac{4}{3 h^{2}} z^{3}\left(\psi(x)+\frac{\partial w(x)}{\partial x}\right) \\
& u_{z}(x, z, t)=w(x)
\end{aligned}
$$


where $u^{0}, w$ and $\psi$ represent the longitudinal displacement, the transverse displacement and the rotation of the cross section at $x$ point, respectively. The strains in the Reddy beam model are defined as:

$\varepsilon_{x x}(x, z, t)=\frac{\partial u^{0}(x)}{\partial x}+z \frac{\partial \psi(x)}{\partial x}-\frac{4}{3 h^{2}} z^{3}\left(\frac{\partial \psi(x)}{\partial x}+\frac{\partial^{2} w(x)}{\partial x^{2}}\right)=\varepsilon_{x x}^{(0)}+z \varepsilon_{x x}^{(1)}+z^{3} \varepsilon_{x x}^{(3)}$

$\gamma_{x z}(x, z, t)=\frac{\partial w(x)}{\partial x}+\psi(x)-\frac{4}{h^{2}} z^{2}\left(\psi(x)+\frac{\partial w(x)}{\partial x}\right)=\gamma_{x z}^{(0)}+z^{2} \gamma_{x z}^{(2)}$

Hamilton's principle is employed to establish the governing equations, expressed by:

$\int_{0}^{t} \delta(U-K+V) d t=0$

where $K$ and $U$ denote the kinetic energy and strain energy respectively, and $V$ indicates the work done by the external forces. The variation of the strain energy can be computed as:

$\delta U=\int_{v}\left(\sigma_{x x} \delta \varepsilon_{x x}+\tau_{x z} \delta \gamma_{x z}\right) d v$

Substituting Eq. (3) into Eq. (5) and, after some simplification, yields:

$\delta U=\int_{0}^{L}\left(Q \delta \varepsilon_{x x}^{(0)}+M \varepsilon_{x x}^{(1)}+E \varepsilon_{x x}^{(3)}+F \gamma_{x z}^{(0)}+R \gamma_{x z}^{(2)}\right) d x$

where

$Q=\int_{A} \sigma_{x x} d A ; \quad M=\int_{A} z \sigma_{x x} d A ; \quad E=\int_{A} z^{3} \sigma_{x x} d A ;$

$F=\int_{A} \tau_{x z} d A ; \quad R=\int_{A} z^{2} \tau_{x z} d A$

The work one by the external forces, $\delta V$, can be evaluated as:

$$
\begin{aligned}
& \delta V=\int_{0}^{L}\left(T(x) \frac{\partial w}{\partial x} \frac{\partial \delta w}{\partial x}+q \delta w+f \delta u^{0}-N \delta \varepsilon_{x x}^{(0)}-\widetilde{M} \frac{\partial \delta \psi}{\partial x}+\frac{4}{3 h^{2}} E \frac{\partial^{2} \delta w}{\partial x^{2}}\right. \\
& \left.-\tilde{F} \delta \gamma_{x z}^{(0)}\right) d x
\end{aligned}
$$

where

$$
\widetilde{M}=M-\frac{4}{3 h^{2}} E ; \quad \tilde{F}=F-\frac{4}{h^{2}} R
$$


Here, $T(x)$ is the external applied force due to the centrifugal loads due to rotation, and $f$ and $q$ indicate the axial and transverse loads. $T(x)$ is calculated as:

$T(x)=b \int_{x}^{L} \int_{-\frac{h}{2}}^{\frac{h}{2}} \rho(x, z) \widetilde{\omega}^{2}(r+x) d z d x$

where $\rho, b, r$ and $\widetilde{\omega}$ are the mass density, the breadth of the beam cross section, the hub radius, and the rotating speed, respectively.

The variation of the virtual kinetic energy for the homogeneous nanobeam is defined as:

$$
\begin{gathered}
\delta K=\int_{0}^{L}\left(J_{0}\left(\frac{\partial u^{0}}{\partial t} \frac{\partial \delta u^{0}}{\partial t}+\frac{\partial w}{\partial t} \frac{\partial \delta w}{\partial t}\right)+J_{1}\left(\frac{\partial u^{0}}{\partial t} \frac{\partial \delta \psi}{\partial t}+\frac{\partial \psi}{\partial t} \frac{\partial \delta}{\partial t}\right)+J_{2} \frac{\partial \psi}{\partial t} \frac{\partial \delta \psi}{\partial t}\right. \\
+\frac{4}{3 h^{2}}\left[-J_{3} \frac{\partial u}{\partial t}\left(\frac{\partial \delta \psi}{\partial t}+\frac{\partial^{2} \delta w}{\partial x \partial t}\right)-J_{3} \frac{\partial \delta u}{\partial t}\left(\frac{\partial \psi}{\partial t}+\frac{\partial^{2} \delta w}{\partial x \partial t}\right)\right. \\
-J_{4} \frac{\partial \psi}{\partial t}\left(\frac{\partial \delta \psi}{\partial t}+\frac{\partial^{2} \delta w}{\partial x \partial t}\right)-J_{4} \frac{\partial \delta \psi}{\partial t}\left(\frac{\partial \psi}{\partial t}+\frac{\partial^{2} w}{\partial x \partial t}\right) \\
\left.\left.+\frac{4}{3 h^{2}} J_{6}\left(\frac{\partial \psi}{\partial t}+\frac{\partial^{2} w}{\partial x \partial t}\right)\left(\frac{\partial \delta \psi}{\partial t}+\frac{\partial^{2} \delta w}{\partial x \partial t}\right)\right]\right) d x
\end{gathered}
$$

where $J_{0}, J_{1}, J_{2}, J_{3}, J_{4}$ and $J_{6}$ are calculated as:

$\left(J_{0}, J_{1}, J_{2}, J_{3}, J_{4}, J_{6}\right)=\int_{A} \rho(x, z)\left(1, z, z^{2}, z^{3}, z^{4}, z^{6}\right) d A$

Substituting Eqs. (7), (9) and (11) into Eq. (4) and setting the coefficients of $\delta u^{0}, \delta w$ and $\delta \psi$ to zero, the equations of motion are obtained as:

$$
\begin{gathered}
\frac{\partial Q}{\partial x}+f-J_{0} \frac{\partial^{2} u^{0}}{\partial t^{2}}-\hat{J}_{1} \frac{\partial^{2} \psi}{\partial t^{2}}+\frac{4}{3 h^{2}} J_{3} \frac{\partial^{3} w}{\partial x \partial t^{2}}=0 \\
\frac{\partial \widetilde{M}}{\partial x}-\tilde{F}-\hat{J}_{1} \frac{\partial^{2} u^{0}}{\partial t^{2}}-\hat{J}_{2} \frac{\partial^{2} \psi}{\partial t^{2}}+\frac{4}{3 h^{2}} \hat{J}_{4}\left(\frac{\partial^{2} \psi}{\partial t^{2}}+\frac{\partial^{3} w}{\partial x \partial t^{2}}\right)=0 \\
\frac{\partial \tilde{F}}{\partial x}+q+\frac{\partial}{\partial x}\left(T(x) \frac{\partial w}{\partial x}\right)+\frac{4}{3 h^{2}} \frac{\partial^{2} E}{\partial x^{2}}-J_{0} \frac{\partial^{2} w}{\partial t^{2}}-\frac{4}{3 h^{2}} J_{3} \frac{\partial^{3} u}{\partial x \partial t^{2}}-\frac{4}{3 h^{2}} J_{4} \frac{\partial^{3} \psi}{\partial x \partial t^{2}} \\
+\left(\frac{4}{3 h^{2}}\right)^{2} J_{6}\left(\frac{\partial^{3} \psi}{\partial x \partial t^{2}}+\frac{\partial^{4} \psi}{\partial x^{2} \partial t^{2}}\right)=0
\end{gathered}
$$

with boundary conditions

$(Q-N) \delta u=0 \quad$ at $x=0, L$

$\widetilde{M} \delta \psi=0 \quad$ at $x=0, L$ 
$\left(\frac{8}{3 h^{2}} \frac{\partial E}{\partial x}+2 \tilde{F}+T(x) \frac{\partial w}{\partial x}-\frac{4}{3 h^{2}} J_{3} \frac{\partial^{2} u}{\partial t^{2}}-\frac{4}{3 h^{2}} \hat{J}_{4} \frac{\partial^{2} \psi}{\partial t^{2}}+\left(\frac{4}{3 h^{2}}\right)^{2} J_{6} \frac{\partial^{3} w}{\partial x \partial t^{2}}\right) \delta w=0$ at $x=0, L$

where $\hat{J}_{1}=J_{1}-\frac{4}{3 h^{2}} J_{3}, \quad \hat{J}_{2}=J_{2}-\frac{4}{3 h^{2}} J_{4}$ and $\hat{J}_{4}=J_{4}-\frac{4}{3 h^{2}} J_{6}$

\section{2-2. GNT Review}

A novel nonlocal theory named GNT was developed by Shaat [4, 42], which is based on the difference between the shear and normal strains in the nonlocal fields. Unlike ENT, GNT utilizes two different nonlocal factors. Based on GNT [4, 42], the nonlocal stress field at each point in the equilibrium equation can be expressed in the following form:

$\sigma_{j i}(\boldsymbol{x})=\int_{V}\left(\lambda \beta_{1}\left(\left|\boldsymbol{x}^{\prime}-\boldsymbol{x}\right|\right) \varepsilon_{r r}\left(\boldsymbol{x}^{\prime}\right) \delta_{i j}+2 \mu \beta_{2}\left(\left|\boldsymbol{x}^{\prime}-\boldsymbol{x}\right|\right) \varepsilon_{i j}\left(\boldsymbol{x}^{\prime}\right)\right) d V^{\prime}$

where $\sigma_{j i}$ indicates the nonlocal stress field that is expressed based on the two different nonlocal factors, $\beta_{1}\left(\left|x^{\prime}-x\right|\right)$ and $\beta_{2}\left(\left|x^{\prime}-x\right|\right)$. Also, $\varepsilon_{i j}=\frac{1}{2}\left(u_{i, j}+u_{j, i}\right)$ defines the nonlocal strain field, and $\lambda$ and $\mu$ are the Lame constants of the material, given in Eq. (1). The equilibrium equations of GNT and classical theory are the same. However, the stress field relevant to GNT is constructed based on the interatomic interactions.

In GNT, to establish the differential form of the constitutive equations of the nonlocal theory, two differential operators are applied as follows [4]:

$\mathcal{L}_{1} \beta_{1}\left(\left|\mathbf{x}^{\prime}-\mathbf{x}\right|\right)=\delta\left(\left|\mathbf{x}^{\prime}-\mathbf{x}\right|\right)$ and $\mathcal{L}_{2} \beta_{2}\left(\left|\mathbf{x}^{\prime}-\mathbf{x}\right|\right)=\delta\left(\left|\mathbf{x}^{\prime}-\mathbf{x}\right|\right)$

where $\delta$ is the Dirac-delta function and $\mathcal{L}_{1}$ and $\mathcal{L}_{2}$ are the differential operators [4]:

$\mathcal{L}_{1}=\left(1-\epsilon_{1} \nabla^{2}\right)$

$\mathcal{L}_{2}=\left(1-\epsilon_{2} \nabla^{2}\right)$

where $\nabla^{2}$ is the Laplacian gradient operator $\left(\nabla^{2}=\frac{\partial^{2}}{\partial x^{2}}\right.$ ). In Eqs. (17) and (18), $\epsilon_{1}$ and $\epsilon_{2}$ denote the constant nonlocal coefficients, which are constrained by $-0.02 a^{2}<\epsilon_{1}<\infty$ and $-0.02 a^{2}<$ $\epsilon_{2}<\infty$, where $a$ is a lattice constant that depends on the crystal structure of the material. The differential equation of the general nonlocal model is then represented by: 


$$
\left(1-\epsilon_{1} \nabla^{2}\right)\left(1-\epsilon_{2} \nabla^{2}\right) \sigma_{(j i)}(\mathbf{x})=\lambda\left(1-\epsilon_{2} \boldsymbol{\nabla}^{2}\right) \varepsilon_{r r}(\mathbf{x}) \delta_{i j}+2 \mu\left(1-\epsilon_{1} \boldsymbol{\nabla}^{2}\right) \varepsilon_{i j}(\mathbf{x})
$$

According to the constitutive equations represented by Eq. (19), the transverse and the longitudinal acoustic dispersions of the material can be considered simultaneously in GNT and this demonstrates the superiority of GNT over Eringen's nonlocal theory [4]. On the basis of GNT presented in Eq. (19), the nonlocal constitutive relations can be expressed as:

$$
\begin{aligned}
& \left(1-\epsilon_{1} \nabla^{2}\right)\left(1-\epsilon_{2} \nabla^{2}\right) \sigma_{x x}=\lambda\left(1-\epsilon_{2} \nabla^{2}\right)\left(\varepsilon_{x x}\right)+2 \mu\left(1-\epsilon_{1} \nabla^{2}\right) \varepsilon_{x x} \\
& \left(1-\epsilon_{1} \nabla^{2}\right)\left(1-\epsilon_{2} \nabla^{2}\right) \sigma_{x y}=2 \mu\left(1-\epsilon_{1} \nabla^{2}\right) \gamma_{x y}
\end{aligned}
$$

To establish the governing equations of motion for rotating nanobeams, the force strain, $N_{i j}$, and the moment strain, $M_{i j}$, are first defined in terms of the displacements, using Eq. (4), as:

$$
\begin{aligned}
\left(1-\epsilon_{1} \nabla^{2}\right)\left(1-\epsilon_{2} \nabla^{2}\right) Q & \\
= & \left(1-\epsilon_{2} \nabla^{2}\right)\left(A_{11} \frac{\partial u}{\partial x}+\left(B_{11}-\frac{4}{3 h^{2}} E_{11}\right) \frac{\partial \psi}{\partial x}-\frac{4}{3 h^{2}} E_{11} \frac{\partial^{2} w}{\partial x^{2}}\right) \\
& +2\left(1-\epsilon_{1} \nabla^{2}\right)\left(\bar{A}_{11} \frac{\partial u}{\partial x}+\left(\bar{B}_{11}-\frac{4}{3 h^{2}} \bar{E}_{11}\right) \frac{\partial \psi}{\partial x}-\frac{4}{3 h^{2}} \bar{E}_{11} \frac{\partial^{2} w}{\partial x^{2}}\right) \\
\left(1-\epsilon_{1} \nabla^{2}\right)\left(1-\epsilon_{2} \nabla^{2}\right) M & \\
= & \left(1-\epsilon_{2} \nabla^{2}\right)\left(B_{11} \frac{\partial u}{\partial x}+\left(D_{11}-\frac{4}{3 h^{2}} F_{11}\right) \frac{\partial \psi}{\partial x}-\frac{4}{3 h^{2}} F_{11} \frac{\partial^{2} w}{\partial x^{2}}\right) \\
& +2\left(1-\epsilon_{1} \nabla^{2}\right)\left(\bar{B}_{11} \frac{\partial u}{\partial x}+\left(\bar{D}_{11}-\frac{4}{3 h^{2}} \bar{F}_{11}\right) \frac{\partial \psi}{\partial x}-\frac{4}{3 h^{2}} \bar{F}_{11} \frac{\partial^{2} w}{\partial x^{2}}\right) \\
\left(1-\epsilon_{1} \nabla^{2}\right)\left(1-\epsilon_{2} \nabla^{2}\right) E & \left(1-\epsilon_{2} \nabla^{2}\right)\left(E_{11} \frac{\partial u}{\partial x}+\left(F_{11}-\frac{4}{3 h^{2}} H_{11}\right) \frac{\partial \psi}{\partial x}-\frac{4}{3 h^{2}} H_{11} \frac{\partial^{2} w}{\partial x^{2}}\right) \\
+ & 2\left(1-\epsilon_{1} \nabla^{2}\right)\left(\bar{E}_{11} \frac{\partial u}{\partial x}+\left(\bar{F}_{11}-\frac{4}{3 h^{2}} \bar{H}_{11}\right) \frac{\partial \psi}{\partial x}-\frac{4}{3 h^{2}} \bar{H}_{11} \frac{\partial^{2} w}{\partial x^{2}}\right) \\
\left(1-\epsilon_{1} \nabla^{2}\right)(1- & \left.\epsilon_{2} \nabla^{2}\right) F=2\left(1-\epsilon_{1} \nabla^{2}\right)\left(A_{12}-\frac{4}{h^{2}} D_{12}\right)\left(\psi+\frac{\partial w}{\partial x}\right) \\
\left(1-\epsilon_{1} \nabla^{2}\right)(1- & \left.\epsilon_{2} \nabla^{2}\right) R=2\left(1-\epsilon_{1} \nabla^{2}\right)\left(D_{12}-\frac{4}{h^{2}} F_{12}\right)\left(\psi+\frac{\partial w}{\partial x}\right)
\end{aligned}
$$

where

$$
\left(A_{11}, B_{11}, D_{11}, E_{11}, F_{11}, H_{11}\right)=\int_{A} \lambda(x, z)\left(1, z, z^{2}, z^{3}, z^{4}, z^{6}\right) d A
$$




$$
\begin{aligned}
& \left(\bar{A}_{11}, \bar{B}_{11}, \bar{D}_{11}, \bar{E}_{11}, \bar{F}_{11}, \bar{H}_{11}\right)=\int_{A} \mu(x, z)\left(1, z, z^{2}, z^{3}, z^{4}, z^{6}\right) d A \\
& \left(A_{12}, D_{12}, F_{12}\right)=\int_{A} \mu(x, z)\left(1, z^{2}, z^{4}\right) d A
\end{aligned}
$$

Based on the definition in Eq. (10), $\widetilde{M}$ and $\widetilde{F}$ can be obtained by combining Eqs. (21-b) and (21-c) and (21-d) and (21-e) respectively as follows:

$$
\begin{aligned}
&\left(1-\epsilon_{1} \nabla^{2}\right)(1-\left.\epsilon_{2} \nabla^{2}\right) \widetilde{M} \\
&=\left(1-\epsilon_{2} \nabla^{2}\right)\left(\alpha_{11} \frac{\partial u}{\partial x}+\beta_{11} \frac{\partial \psi}{\partial x}-\frac{4}{3 h^{2}} \delta_{11}\left(\frac{\partial \psi}{\partial x}+\frac{\partial^{2} w}{\partial x^{2}}\right)\right) \\
&+ 2\left(1-\epsilon_{1} \nabla^{2}\right)\left(\bar{\alpha}_{11} \frac{\partial u}{\partial x}+\bar{\beta}_{11} \frac{\partial \psi}{\partial x}-\frac{4}{3 h^{2}} \bar{\delta}_{11}\left(\frac{\partial \psi}{\partial x}+\frac{\partial^{2} w}{\partial x^{2}}\right)\right) \\
&\left(1-\epsilon_{1} \nabla^{2}\right)\left(1-\epsilon_{2} \nabla^{2}\right) \tilde{F}=2 \mu\left(1-\epsilon_{1} \nabla^{2}\right) \bar{A}\left(\psi+\frac{\partial w}{\partial x}\right)
\end{aligned}
$$

where

$$
\begin{aligned}
& \alpha_{11}=B_{11}-\frac{4}{3 h^{2}} E_{11}, \quad \beta_{11}=D_{11}-\frac{4}{3 h^{2}} F_{11}, \quad \delta_{11}=F_{11}-\frac{4}{3 h^{2}} H_{11} \\
& \bar{\alpha}_{11}=\bar{B}_{11}-\frac{4}{3 h^{2}} \bar{E}_{11}, \quad \bar{\beta}_{11}=\bar{D}_{11}-\frac{4}{3 h^{2}} \bar{F}_{11}, \quad \bar{\delta}_{11}=\bar{F}_{11}-\frac{4}{3 h^{2}} \bar{H}_{11} \\
& \bar{A}=A_{12}-\frac{4}{h^{2}} D_{12}-\frac{4}{h^{2}} D_{12}+\left(\frac{4}{h^{2}}\right)^{2} F_{12}
\end{aligned}
$$

Then, by multiplying Eq. (14) by $\left(1-\epsilon_{1} \nabla^{2}\right)\left(1-\epsilon_{2} \nabla^{2}\right)$ and utilizing Eqs. (21) and (23), the governing equations of motion of the rotating nonlocal beam are obtained in terms of the displacements as:

$$
\begin{aligned}
\left(1-\epsilon_{2} \nabla^{2}\right)\left(A_{11}\right. & \left.\frac{\partial^{2} u}{\partial x^{2}}+\left(B_{11}-\frac{4}{3 h^{2}} E_{11}\right) \frac{\partial^{2} \psi}{\partial x^{2}}-\frac{4}{3 h^{2}} E_{11} \frac{\partial^{3} w}{\partial x^{3}}\right) \\
+ & 2\left(1-\epsilon_{1} \nabla^{2}\right)\left(\bar{A}_{11} \frac{\partial^{2} u}{\partial x^{2}}+\left(\bar{B}_{11}-\frac{4}{3 h^{2}} \bar{E}_{11}\right) \frac{\partial^{2} \psi}{\partial x^{2}}-\frac{4}{3 h^{2}} \bar{E}_{11} \frac{\partial^{3} w}{\partial x^{3}}\right) \\
& -\left(1-\epsilon_{1} \nabla^{2}\right)\left(1-\epsilon_{2} \nabla^{2}\right)\left(-f+J_{0} \frac{\partial^{2} u^{0}}{\partial t^{2}}+\hat{J}_{1} \frac{\partial^{2} \psi}{\partial t^{2}}-\frac{4}{3 h^{2}} J_{3} \frac{\partial^{3} w}{\partial x \partial t^{2}}\right)=0
\end{aligned}
$$




$$
\begin{aligned}
\left(1-\epsilon_{2} \nabla^{2}\right)\left(\alpha_{11}\right. & \left.\frac{\partial^{2} u}{\partial x^{2}}+\beta_{11} \frac{\partial^{2} \psi}{\partial x^{2}}-\frac{4}{3 h^{2}} \delta_{11}\left(\frac{\partial^{2} \psi}{\partial x^{2}}+\frac{\partial^{3} w}{\partial x^{3}}\right)\right) \\
& +2\left(1-\epsilon_{1} \nabla^{2}\right)\left(\bar{\alpha}_{11} \frac{\partial u}{\partial x}+\bar{\beta}_{11} \frac{\partial \psi}{\partial x}-\frac{4}{3 h^{2}} \bar{\delta}_{11}\left(\frac{\partial^{2} \psi}{\partial x^{2}}+\frac{\partial^{3} w}{\partial x^{3}}\right)\right) \\
& -2\left(1-\epsilon_{1} \nabla^{2}\right) \bar{A}\left(\psi+\frac{\partial w}{\partial x}\right) \\
& -\left(1-\epsilon_{1} \nabla^{2}\right)\left(1-\epsilon_{2} \nabla^{2}\right)\left(\hat{J}_{1} \frac{\partial^{2} u^{0}}{\partial t^{2}}+\hat{J}_{2} \frac{\partial^{2} \psi}{\partial t^{2}}-\frac{4}{3 h^{2}} \hat{J}_{4}\left(\frac{\partial^{2} \psi}{\partial t^{2}}+\frac{\partial^{3} w}{\partial x \partial t^{2}}\right)\right) \\
= & 0 \\
2\left(1-\epsilon_{1} \nabla^{2}\right) \bar{A}( & \left.\frac{\partial \psi}{\partial x}+\frac{\partial^{2} w}{\partial x^{2}}\right) \\
& +\frac{4}{3 h^{2}}\left[\left(1-\epsilon_{2} \nabla^{2}\right)\left(E_{11} \frac{\partial^{3} u}{\partial x^{3}}+\left(F_{11}-\frac{4}{3 h^{2}} H_{11}\right) \frac{\partial^{3} \psi}{\partial x^{3}}-\frac{4}{3 h^{2}} H_{11} \frac{\partial^{4} w}{\partial x^{4}}\right)\right. \\
& \left.+2\left(1-\epsilon_{1} \nabla^{2}\right)\left(\bar{E}_{11} \frac{\partial^{3} u}{\partial x^{3}}+\left(\bar{F}_{11}-\frac{4}{3 h^{2}} \bar{H}_{11}\right) \frac{\partial^{3} \psi}{\partial x^{3}}-\frac{4}{3 h^{2}} \bar{H}_{11} \frac{\partial^{4} w}{\partial x^{4}}\right)\right] \\
& \left.-\left(\frac{4}{3 h^{2}}\right) J_{6}\left(\frac{\partial^{3} \psi}{\partial x \partial t^{2}}+\frac{\partial^{4} \psi}{\partial x^{2} \partial t^{2}}\right)\right)=0 \\
& +\left(1-\epsilon_{1} \nabla^{2}\right)\left(1-\epsilon_{2} \nabla^{2}\right)\left[q+\frac{\partial}{\partial x}\left(T(x) \frac{\partial w}{\partial x}\right)\right] \\
& \left(1-\epsilon_{1} \nabla^{2}\right)\left(1-\epsilon_{2} \nabla^{2}\right)\left(J_{0} \frac{\partial^{2} w}{\partial t^{2}}+\frac{4}{3 h^{2}} J_{3} \frac{\partial^{3} u}{\partial x \partial t^{2}}+\frac{4}{3 h^{2}} J_{4} \frac{\partial^{3} \psi}{\partial x \partial t^{2}}\right. \\
& \\
& \\
&
\end{aligned}
$$

Equations (25) to (27) can be rewritten as: 


$$
\begin{aligned}
& \left(-\varepsilon_{2} \alpha_{11}-2 \varepsilon_{1} \bar{\alpha}_{11}\right) \frac{\partial^{4} u}{\partial x^{4}}+\left(-2 \varepsilon_{2} \frac{\partial \alpha_{11}}{\partial x}-4 \varepsilon_{1} \frac{\partial \bar{\alpha}_{11}}{\partial x}\right) \frac{\partial^{3} u}{\partial x^{3}}+\left(\alpha_{11}+2 \bar{\alpha}_{11}-\varepsilon_{2} \frac{\partial^{2} \alpha_{11}}{\partial x^{2}}-2 \varepsilon_{1} \frac{\partial^{2} \bar{\alpha}_{11}}{\partial x^{2}}\right) \frac{\partial^{2} u}{\partial x^{2}}+ \\
& \left(-\varepsilon_{2} \zeta_{11}-2 \varepsilon_{1} \bar{\zeta}_{11}\right) \frac{\partial^{4} \psi}{\partial x^{4}}+\left(-2 \varepsilon_{2} \frac{\partial \zeta_{11}}{\partial x}-4 \varepsilon_{1} \frac{\partial \bar{\zeta}_{11}}{\partial x}\right) \frac{\partial^{3} \psi}{\partial x^{3}}+\left(\zeta_{11}+2 \bar{\zeta}_{11}-\varepsilon_{2} \frac{\partial^{2} \zeta_{11}}{\partial x^{2}}-2 \varepsilon_{1} \frac{\partial^{2} \bar{\zeta}_{11}}{\partial x^{2}}+2 \varepsilon_{1} \bar{A}\right) \frac{\partial^{2} \psi}{\partial x^{2}}+ \\
& \left(4 \varepsilon_{1} \frac{\partial \bar{A}}{\partial x}\right) \frac{\partial \psi}{\partial x}+\left(-2 \bar{A}+2 \varepsilon_{1} \frac{\partial^{2} \bar{A}}{\partial x^{2}}\right) \psi+\bar{\kappa}\left(-\varepsilon_{2} \delta_{11}-2 \varepsilon_{1} \bar{\delta}_{11}\right) \frac{\partial^{5} w}{\partial x^{5}}+\bar{\kappa}\left(-2 \varepsilon_{2} \frac{\partial \delta_{11}}{\partial x}-4 \varepsilon_{1} \frac{\partial \bar{\delta}_{11}}{\partial x}\right) \frac{\partial^{4} w}{\partial x^{4}} \\
& {\left[\bar{\kappa}\left(\delta_{11}+2 \bar{\delta}_{11}-\varepsilon_{2} \frac{\partial^{2} \delta_{11}}{\partial x^{2}}-2 \varepsilon_{1} \frac{\partial^{2} \bar{\delta}_{11}}{\partial x^{2}}\right)+2 \varepsilon_{1} \bar{A}\right] \frac{\partial^{3} w}{\partial x^{3}}+\left(4 \varepsilon_{1} \frac{\partial \bar{A}}{\partial x}\right) \frac{\partial^{2} w}{\partial x^{2}}+\left(-2 \bar{A}+2 \varepsilon_{1} \frac{\partial^{2} \bar{A}}{\partial x^{2}}\right) \frac{\partial w}{\partial x}+} \\
& {\left[-\hat{J}_{1}+\left(\varepsilon_{1}+\varepsilon_{2}\right) \frac{\partial^{2} \hat{J}_{1}}{\partial x^{2}}-\varepsilon_{1} \varepsilon_{2} \frac{\partial^{4} \hat{J}_{1}}{\partial x^{4}}\right] \frac{\partial^{2} u}{\partial t^{2}}+\left[2\left(\varepsilon_{1}+\varepsilon_{2}\right) \frac{\partial \hat{J}_{1}}{\partial x}-4 \varepsilon_{1} \varepsilon_{2} \frac{\partial^{3} \hat{J}_{1}}{\partial x^{3}}\right] \frac{\partial^{3} u}{\partial x \partial t^{2}}+} \\
& {\left[\left(\varepsilon_{1}+\varepsilon_{2}\right) \hat{J}_{1}-6 \varepsilon_{1} \varepsilon_{2} \frac{\partial^{2} \hat{J}_{1}}{\partial x^{2}}\right] \frac{\partial^{4} u}{\partial x^{2} \partial t^{2}}+\left[-4 \varepsilon_{1} \varepsilon_{2} \frac{\partial \hat{J}_{1}}{\partial x}\right] \frac{\partial^{5} u}{\partial x^{3} \partial t^{2}}+\left[-\varepsilon_{1} \varepsilon_{2} \hat{J}_{1}\right] \frac{\partial^{6} u}{\partial x^{4} \partial t^{2}}+} \\
& {\left[-\bar{J}_{2}+\left(\varepsilon_{1}+\varepsilon_{2}\right) \frac{\partial^{2} \bar{J}_{2}}{\partial x^{2}}-\varepsilon_{1} \varepsilon_{2} \frac{\partial^{4} \bar{J}_{2}}{\partial x^{4}}\right] \frac{\partial^{2} \psi}{\partial t^{2}}+\left[2\left(\varepsilon_{1}+\varepsilon_{2}\right) \frac{\partial \bar{J}_{2}}{\partial x}-4 \varepsilon_{1} \varepsilon_{2} \frac{\partial^{3} \bar{J}_{2}}{\partial x^{3}}\right] \frac{\partial^{3} \psi}{\partial x \partial t^{2}}+} \\
& {\left[\left(\varepsilon_{1}+\varepsilon_{2}\right) \bar{J}_{2}-6 \varepsilon_{1} \varepsilon_{2} \frac{\partial^{2} \bar{J}_{2}}{\partial x^{2}}\right] \frac{\partial^{4} \psi}{\partial x^{2} \partial t^{2}}+\left[-4 \varepsilon_{1} \varepsilon_{2} \frac{\partial \bar{J}_{2}}{\partial x}\right] \frac{\partial^{5} \psi}{\partial x^{3} \partial t^{2}}+\left[-\varepsilon_{1} \varepsilon_{2} \bar{J}_{2}\right] \frac{\partial^{6} \psi}{\partial x^{4} \partial t^{2}}+} \\
& \bar{\kappa}\left[-\hat{J}_{4}+\left(\varepsilon_{1}+\varepsilon_{2}\right) \frac{\partial^{2} \hat{J}_{4}}{\partial x^{2}}-\varepsilon_{1} \varepsilon_{2} \frac{\partial^{4} \hat{J}_{4}}{\partial x^{4}}\right] \frac{\partial^{3} w}{\partial x \partial t^{2}}+\bar{\kappa}\left[2\left(\varepsilon_{1}+\varepsilon_{2}\right) \frac{\partial \hat{J}_{4}}{\partial x}-4 \varepsilon_{1} \varepsilon_{2} \frac{\partial^{3} \hat{J}_{4}}{\partial x^{3}}\right] \frac{\partial^{4} w}{\partial x^{2} \partial t^{2}}+ \\
& \bar{\kappa}\left[\left(\varepsilon_{1}+\varepsilon_{2}\right) \hat{J}_{4}-6 \varepsilon_{1} \varepsilon_{2} \frac{\partial^{2} \hat{J}_{4}}{\partial x^{2}}\right] \frac{\partial^{5} w}{\partial x^{3} \partial t^{2}}+\left[-4 \varepsilon_{1} \varepsilon_{2} \bar{\kappa} \frac{\partial \hat{J}_{4}}{\partial x}\right] \frac{\partial^{6} w}{\partial x^{4} \partial t^{2}}+\left[-\varepsilon_{1} \varepsilon_{2} \bar{\kappa} \hat{J}_{4}\right] \frac{\partial^{7} w}{\partial x^{5} \partial t^{2}}=0
\end{aligned}
$$




$$
\begin{aligned}
& \bar{\kappa}\left(\varepsilon_{2} E_{11}+2 \varepsilon_{1} \bar{E}_{11}\right) \frac{\partial^{5} u}{\partial x^{5}}+\bar{\kappa}\left(2 \varepsilon_{2} \frac{\partial E_{11}}{\partial x}+4 \varepsilon_{1} \frac{\partial \bar{E}_{11}}{\partial x}\right) \frac{\partial^{4} u}{\partial x^{4}}+\bar{\kappa}\left(-E_{11}-2 \bar{E}_{11}+\varepsilon_{2} \frac{\partial^{2} E_{11}}{\partial x^{2}}+2 \varepsilon_{1} \frac{\partial^{2} \bar{E}_{11}}{\partial x^{2}}\right) \frac{\partial^{3} u}{\partial x^{3}}+ \\
& \bar{\kappa}\left(\varepsilon_{2} \delta_{11}+2 \varepsilon_{1} \bar{\delta}_{11}\right) \frac{\partial^{5} \psi}{\partial x^{5}}+\bar{\kappa}\left(2 \varepsilon_{2} \frac{\partial \delta_{11}}{\partial x}+4 \varepsilon_{1} \frac{\partial \bar{\delta}_{11}}{\partial x}\right) \frac{\partial^{4} \psi}{\partial x^{4}}+\left[-\bar{\kappa}\left(\delta_{11}+2 \bar{\delta}_{11}+\varepsilon_{2} \frac{\partial^{2} \delta_{11}}{\partial x^{2}}+2 \varepsilon_{1} \frac{\partial^{2} \bar{\delta}_{11}}{\partial x^{2}}\right)-2 \varepsilon_{1} \bar{A}\right] \frac{\partial^{3} \psi}{\partial x^{3}}+ \\
& \left(-4 \varepsilon_{1} \frac{\partial \bar{A}}{\partial x}\right) \frac{\partial^{2} \psi}{\partial x^{2}}+\left(2 \bar{A}-2 \varepsilon_{1} \frac{\partial^{2} \bar{A}}{\partial x^{2}}\right) \frac{\partial \psi}{\partial x}+\left[\bar{\kappa}^{2}\left(\varepsilon_{2} H_{11}+2 \varepsilon_{1} \bar{H}_{11}\right)+\varepsilon_{1} \varepsilon_{2} T\right] \frac{\partial^{6} w}{\partial x^{6}}+\left[\bar{\kappa}^{2}\left(2 \varepsilon_{2} \frac{\partial H_{11}}{\partial x}+4 \varepsilon_{1} \frac{\partial \bar{H}_{11}}{\partial x}\right)+\right. \\
& \left.5 \varepsilon_{1} \varepsilon_{2} \frac{\partial T}{\partial x}\right] \frac{\partial^{5} w}{\partial x^{5}}+\left[-\bar{\kappa}^{2}\left(H_{11}+2 \bar{H}_{11}+\varepsilon_{2} \frac{\partial^{2} H_{11}}{\partial x^{2}}+2 \varepsilon_{1} \frac{\partial^{2} \bar{H}_{11}}{\partial x^{2}}\right)-2 \varepsilon_{1} \bar{A}-\left(\varepsilon_{1}+\varepsilon_{2}\right) T+10 \varepsilon_{1} \varepsilon_{2} \frac{\partial^{2} T}{\partial x^{2}}\right] \frac{\partial^{4} w}{\partial x^{4}}+ \\
& {\left[-4 \varepsilon_{1} \frac{\partial \bar{A}}{\partial x}-3\left(\varepsilon_{1}+\varepsilon_{2}\right) \frac{\partial T}{\partial x}\right] \frac{\partial^{3} w}{\partial x^{3}}+\left[2 \bar{A}-2 \varepsilon_{1} \frac{\partial^{2} \bar{A}}{\partial x^{2}}+T-3\left(\varepsilon_{1}+\varepsilon_{2}\right) \frac{\partial^{2} T}{\partial x^{2}}\right] \frac{\partial^{2} w}{\partial x^{2}}+\left(\frac{\partial T}{\partial x}\right) \frac{\partial w}{\partial x}+} \\
& \bar{\kappa}\left[+J_{3}-\left(\varepsilon_{1}+\varepsilon_{2}\right) \frac{\partial^{2} J_{3}}{\partial x^{2}}+\varepsilon_{1} \varepsilon_{2} \frac{\partial^{4} J_{3}}{\partial x^{4}}\right] \frac{\partial^{3} u}{\partial x \partial t^{2}}+\bar{\kappa}\left[-2\left(\varepsilon_{1}+\varepsilon_{2}\right) \frac{\partial J_{3}}{\partial x}+4 \varepsilon_{1} \varepsilon_{2} \frac{\partial^{3} J_{3}}{\partial x^{3}}\right] \frac{\partial^{4} u}{\partial x^{2} \partial t^{2}}+ \\
& \bar{\kappa}\left[-\left(\varepsilon_{1}+\varepsilon_{2}\right) J_{3}+6 \varepsilon_{1} \varepsilon_{2} \frac{\partial^{2} J_{3}}{\partial x^{2}}\right] \frac{\partial^{5} u}{\partial x^{3} \partial t^{2}}+\left[4 \varepsilon_{1} \varepsilon_{2} \bar{\kappa} \frac{\partial J_{3}}{\partial x}\right] \frac{\partial^{6} u}{\partial x^{4} \partial t^{2}}+\left[\varepsilon_{1} \varepsilon_{2} \bar{\kappa} J_{3}\right] \frac{\partial^{7} u}{\partial x^{5} \partial t^{2}}+ \\
& \bar{\kappa}\left[+\hat{J}_{4}-\left(\varepsilon_{1}+\varepsilon_{2}\right) \frac{\partial^{2} \hat{J}_{4}}{\partial x^{2}}+\varepsilon_{1} \varepsilon_{2} \frac{\partial^{4} \hat{J}_{4}}{\partial x^{4}}\right] \frac{\partial^{3} \psi}{\partial x \partial t^{2}}+\bar{\kappa}\left[-2\left(\varepsilon_{1}+\varepsilon_{2}\right) \frac{\partial \hat{J}_{4}}{\partial x}+4 \varepsilon_{1} \varepsilon_{2} \frac{\partial^{3} \hat{J}_{4}}{\partial x^{3}}\right] \frac{\partial^{4} \psi}{\partial x^{2} \partial t^{2}}+ \\
& \bar{\kappa}\left[-\left(\varepsilon_{1}+\varepsilon_{2}\right) \hat{J}_{4}+6 \varepsilon_{1} \varepsilon_{2} \frac{\partial^{2} \hat{J}_{4}}{\partial x^{2}}\right] \frac{\partial^{5} \psi}{\partial x^{3} \partial t^{2}}+\left[4 \varepsilon_{1} \varepsilon_{2} \bar{\kappa} \frac{\partial \hat{J}_{4}}{\partial x}\right] \frac{\partial^{6} \psi}{\partial x^{4} \partial t^{2}}+\left[\varepsilon_{1} \varepsilon_{2} \bar{\kappa} \hat{J}_{4}\right] \frac{\partial^{7} \psi}{\partial x^{5} \partial t^{2}} \\
& {\left[-J_{0}+\left(\varepsilon_{1}+\varepsilon_{2}\right) \frac{\partial^{2} J_{0}}{\partial x^{2}}-\varepsilon_{1} \varepsilon_{2} \frac{\partial^{4} J_{0}}{\partial x^{4}}\right] \frac{\partial^{2} w}{\partial t^{2}}+\left[2\left(\varepsilon_{1}+\varepsilon_{2}\right) \frac{\partial J_{0}}{\partial x}-4 \varepsilon_{1} \varepsilon_{2} \frac{\partial^{3} J_{0}}{\partial x^{3}}\right] \frac{\partial^{3} w}{\partial x \partial t^{2}}+} \\
& {\left[\bar{\kappa}^{2} J_{6}+\left(\varepsilon_{1}+\varepsilon_{2}\right) J_{0}-\bar{\kappa}^{2}\left(\varepsilon_{1}+\varepsilon_{2}\right) \frac{\partial^{2} J_{6}}{\partial x^{2}}-6 \varepsilon_{1} \varepsilon_{2} \frac{\partial^{2} J_{0}}{\partial x^{2}}+\varepsilon_{1} \varepsilon_{2} \bar{\kappa}^{2} \frac{\partial^{4} J_{6}}{\partial x^{4}}\right] \frac{\partial^{4} w}{\partial x^{2} \partial t^{2}}+} \\
& {\left[-2 \bar{\kappa}^{2}\left(\varepsilon_{1}+\varepsilon_{2}\right) \frac{\partial J_{6}}{\partial x}-4 \varepsilon_{1} \varepsilon_{2} \frac{\partial J_{0}}{\partial x}+4 \varepsilon_{1} \varepsilon_{2} \bar{\kappa}^{2} \frac{\partial^{3} J_{6}}{\partial x^{3}}\right] \frac{\partial^{5} w}{\partial x^{3} \partial t^{2}}+} \\
& {\left[-\bar{\kappa}^{2}\left(\varepsilon_{1}+\varepsilon_{2}\right) J_{6}-\varepsilon_{1} \varepsilon_{2} J_{0}+6 \varepsilon_{1} \varepsilon_{2} \bar{\kappa}^{2} \frac{\partial^{2} J_{6}}{\partial x^{2}}\right] \frac{\partial^{6} w}{\partial x^{4} \partial t^{2}}+\left[4 \varepsilon_{1} \varepsilon_{2} \bar{\kappa}^{2} \frac{\partial J_{6}}{\partial x}\right] \frac{\partial^{7} w}{\partial x^{5} \partial t^{2}}+\left[\varepsilon_{1} \varepsilon_{2} \bar{\kappa}^{2} J_{6}\right] \frac{\partial^{8} w}{\partial x^{6} \partial t^{2}}=0}
\end{aligned}
$$

where

$\zeta_{11}=\beta_{11}+\bar{\kappa} \delta_{11}$

$\bar{\zeta}_{11}=\bar{\beta}_{11}+\bar{\kappa} \bar{\delta}_{11}$

$\bar{J}_{2}=\hat{J}_{2}+\bar{\kappa} \hat{J}_{4}$

$\bar{\kappa}=\frac{-4}{3 h^{2}}$

It should be noted that in a 2D-FG nano beam, the material configuration alters along both the longitudinal and the thickness directions. The nonlocal parameters $\epsilon_{1}$ and $\epsilon_{2}$ also alter along longitudinal (x-axis) and thickness (z-axis) directions, similarly to the other mechanical properties, as:

$$
\begin{aligned}
& \epsilon_{1}(x, z)=\left(\epsilon_{1}\right)_{m}+\left[\left(\epsilon_{1}\right)_{c}-\left(\epsilon_{1}\right)_{m}\right]\left(\frac{1}{2}+\frac{z}{h}\right)^{n z}\left(\frac{x}{L}\right)^{n x}-\frac{\gamma}{2}\left[\left(\epsilon_{1}\right)_{c}+\left(\epsilon_{1}\right)_{m}\right] \\
& \epsilon_{2}(x, z)=\left(\epsilon_{2}\right)_{m}+\left[\left(\epsilon_{2}\right)_{c}-\left(\epsilon_{2}\right)_{m}\right]\left(\frac{1}{2}+\frac{z}{h}\right)^{n z}\left(\frac{x}{L}\right)^{n x}-\frac{\gamma}{2}\left[\left(\epsilon_{2}\right)_{c}+\left(\epsilon_{2}\right)_{m}\right]
\end{aligned}
$$


where the ()$_{m}$ and ()$_{c}$ subscripts denote the metal and ceramic properties respectively.

\section{Solution Procedure}

\subsection{Generalized Differential Quadrature Method}

The GDQM is an efficient and robust numerical solution technique, and hence is adopted to solve the equations of motion of the 2D-FG rotating nanobeam and to extract the results. Based on GDQM, the domain of the nanobeam is discretized into $N_{x}$ mesh points in the $x$ direction. The mesh points are generated by the Chebyshev-Gauss-Lobatto distribution to give:

$x_{i}=\frac{1}{2}\left(1-\cos \left(\frac{i-1}{N_{x}-1} \pi\right)\right) i=1, \ldots, N_{x}$

To implement the GDQM, all of the derivatives of $f(x)$ at each mesh point $x_{i}$ are defined via a linear weighted sum as:

$\frac{\partial^{n} f(x)}{\partial x^{n}} \mid\left(x_{i}\right)=\sum_{k=1}^{N_{x}} \Gamma_{i k}^{x(n)} f\left(x_{k}\right)$

where $i=1, \ldots, N_{x}$ and $\Gamma_{i k}^{x(n)}$ are weighting coefficients corresponding to the $n^{t \square}$ order derivative with respect to $x$. The weighting coefficients for the first order derivative are:

$\Gamma_{i k}^{x(1)}=\left\{\begin{array}{l}\frac{r\left(x_{i}\right)}{\left(x_{i}-x_{k}\right) r\left(x_{k}\right)} \text { for } i \neq k \\ -\sum_{\substack{k=1 \\ N_{x}}}^{N_{i \neq k}} \Gamma_{i k}^{x(1)} \text { for } i=k\end{array}\right.$

where $r\left(x_{i}\right)$ are the Lagrangian polynomials given by:

$r\left(x_{i}\right)=\prod_{\substack{k=1 \\ i \neq k}}^{N_{x}}\left(x_{i}-x_{k}\right)$

The weighting coefficients for higher order derivatives can be calculated as [45]:

$\Gamma_{i k}^{(n)}=\sum_{p=1}^{N_{x}} \Gamma_{i p}^{(n-1)} \Gamma_{p k}^{(1)}$

The solution of the equations of motion are assumed to be of the form:

$u(x, t)=U(x) \exp (j \omega t)$

$w(x, t)=W(x) \exp (j \omega t)$

$\psi(x, t)=\Psi(x) \exp (j \omega t)$

where $\omega$ is the natural frequency and $j=\sqrt{-1}$. Using GDQM principles, the governing equations of motion (Eqs. (28) to (30)) are discretized to give the following equations: 


$$
\begin{gathered}
\left(-\varepsilon_{2} A_{11}-2 \varepsilon_{1} \bar{A}_{11}\right)_{i} \sum_{k=1}^{N_{x}} \Gamma_{i k}^{(4)} U_{k}+\left(\sum_{k=1}^{N_{x}} \Gamma_{i k}^{(1)}\left(-2 \varepsilon_{2} A_{11}-4 \varepsilon_{1} \bar{A}_{11}\right)_{k}\right) \sum_{k=1}^{N_{x}} \Gamma_{i k}^{(3)} U_{k}+ \\
\left(\left(A_{11}+2 \bar{A}_{11}\right)_{i}+\sum_{k=1}^{N_{x}} \Gamma_{i k}^{(2)}\left(-\varepsilon_{2} A_{11}-2 \varepsilon_{1} \bar{A}_{11}\right)_{k}\right) \sum_{k=1}^{N_{x}} \Gamma_{i k}^{(2)} U_{k}+\left(-\varepsilon_{2} \alpha_{11}-2 \varepsilon_{1} \bar{\alpha}_{11}\right)_{i} \sum_{k=1}^{N_{x}} \Gamma_{i k}^{(4)} \Psi_{k}+ \\
\left(\sum_{k=1}^{N_{x}} \Gamma_{i k}^{(1)}\left(-2 \varepsilon_{2} \alpha_{11}-4 \varepsilon_{1} \bar{\alpha}_{11}\right)_{k}\right) \sum_{k=1}^{N_{x}} \Gamma_{i k}^{(3)} \Psi_{k} \\
+\left(\left(\alpha_{11}+2 \bar{\alpha}_{11}\right)_{i}+\sum_{k=1}^{N_{x}} \Gamma_{i k}^{(2)}\left(-\varepsilon_{2} \alpha_{11}-2 \varepsilon_{1} \bar{\alpha}_{11}\right)_{k}\right) \sum_{k=1}^{N_{x}} \Gamma_{i k}^{(2)} \Psi_{k}+
\end{gathered}
$$$$
\bar{\kappa}\left(-\varepsilon_{2} E_{11}-2 \varepsilon_{1} \bar{E}_{11}\right)_{i} \sum_{k=1}^{N_{x}} \Gamma_{i k}^{(5)} W_{k}+\bar{\kappa}\left(\sum_{k=1}^{N_{x}} \Gamma_{i k}^{(1)}\left(-2 \varepsilon_{2} E_{11}-4 \varepsilon_{1} \bar{E}_{11}\right)_{k}\right) \sum_{k=1}^{N_{x}} \Gamma_{i k}^{(4)} W_{k}+
$$$$
\bar{\kappa}\left(\left(E_{11}+2 \bar{E}_{11}\right)_{i}+\sum_{k=1}^{N_{x}} \Gamma_{i k}^{(2)}\left(-\varepsilon_{2} E_{11}-2 \varepsilon_{1} \bar{E}_{11}\right)_{k}\right) \sum_{k=1}^{N_{x}} \Gamma_{i k}^{(3)} W_{k}+
$$$$
\omega^{2}\left\{\left(\left(-J_{0}\right)_{i}+\left(\varepsilon_{1}+\varepsilon_{2}\right) \sum_{k=1}^{N_{x}} \Gamma_{i k}^{(2)}\left(J_{0}\right)_{k}-\varepsilon_{1} \varepsilon_{2} \sum_{k=1}^{N_{x}} \Gamma_{i k}^{(4)}\left(J_{0}\right)_{k}\right) U_{i}+\right.
$$$$
\left(2\left(\varepsilon_{1}+\varepsilon_{2}\right) \sum_{k=1}^{N_{x}} \Gamma_{i k}^{(1)}\left(J_{0}\right)_{k}-4 \varepsilon_{1} \varepsilon_{2} \sum_{k=1}^{N_{x}} \Gamma_{i k}^{(3)}\left(J_{0}\right)_{k}\right) \sum_{k=1}^{N_{x}} \Gamma_{i k}^{(1)} U_{k}
$$$$
+\left(\left(\varepsilon_{1}+\varepsilon_{2}\right)\left(J_{0}\right)_{i}-6 \varepsilon_{1} \varepsilon_{2} \sum_{k=1}^{N_{x}} \Gamma_{i k}^{(2)}\left(J_{0}\right)_{k}\right) \sum_{k=1}^{N_{x}} \Gamma_{i k}^{(2)} U_{k}
$$$$
+\left(-4 \varepsilon_{1} \varepsilon_{2} \sum_{k=1}^{N_{x}} \Gamma_{i k}^{(1)}\left(J_{0}\right)_{k}\right) \sum_{k=1}^{N_{x}} \Gamma_{i k}^{(3)} U_{k}+\left(-\varepsilon_{1} \varepsilon_{2}\left(J_{0}\right)_{i}\right) \sum_{k=1}^{N_{x}} \Gamma_{i k}^{(4)} U_{k}+
$$$$
\left(\left(-\hat{J}_{1}\right)_{i}+\left(\varepsilon_{1}+\varepsilon_{2}\right) \sum_{k=1}^{N_{x}} \Gamma_{i k}^{(2)}\left(\hat{J}_{1}\right)_{k}-\varepsilon_{1} \varepsilon_{2} \sum_{k=1}^{N_{x}} \Gamma_{i k}^{(4)}\left(\hat{J}_{1}\right)_{k}\right) \Psi_{k}
$$$$
+\left(2\left(\varepsilon_{1}+\varepsilon_{2}\right) \sum_{k=1}^{N_{x}} \Gamma_{i k}^{(1)}\left(\hat{J}_{1}\right)_{k}-4 \varepsilon_{1} \varepsilon_{2} \sum_{k=1}^{N_{x}} \Gamma_{i k}^{(3)}\left(\hat{J}_{1}\right)_{k}\right)
$$ 


$$
\begin{aligned}
& \sum_{k=1}^{N_{x}} \Gamma_{i k}^{(1)} \Psi_{k}+\left(\left(\varepsilon_{1}+\varepsilon_{2}\right)\left(\hat{J}_{1}\right)_{i}-6 \varepsilon_{1} \varepsilon_{2} \sum_{k=1}^{N_{x}} \Gamma_{i k}^{(2)}\left(\hat{J}_{1}\right)_{k}\right) \sum_{k=1}^{N_{x}} \Gamma_{i k}^{(2)} \Psi_{k} \\
& +\left(-4 \varepsilon_{1} \varepsilon_{2} \sum_{k=1}^{N_{x}} \Gamma_{i k}^{(1)}\left(\hat{U}_{1}\right)_{k}\right) \sum_{k=1}^{N_{x}} \Gamma_{i k}^{(3)} \Psi_{k}+ \\
& \left(-\varepsilon_{1} \varepsilon_{2}\left(\hat{J}_{1}\right)_{i}\right) \sum_{k=1}^{N_{x}} \Gamma_{i k}^{(4)} \Psi_{k}+\bar{\kappa}\left(\left(-J_{3}\right)_{i}+\left(\varepsilon_{1}+\varepsilon_{2}\right) \sum_{k=1}^{N_{x}} \Gamma_{i k}^{(2)}\left(J_{3}\right)_{k}-\varepsilon_{1} \varepsilon_{2} \sum_{k=1}^{N_{x}} \Gamma_{i k}^{(4)}\left(J_{3}\right)_{k}\right) \sum_{k=1}^{N_{x}} \Gamma_{i k}^{(1)} W_{k}+ \\
& \bar{\kappa}\left(2\left(\varepsilon_{1}+\varepsilon_{2}\right) \sum_{k=1}^{N_{x}} \Gamma_{i k}^{(1)}\left(J_{3}\right)_{k}-4 \varepsilon_{1} \varepsilon_{2} \sum_{k=1}^{N_{x}} \Gamma_{i k}^{(3)}\left(J_{3}\right)_{k}\right) \sum_{k=1}^{N_{x}} \Gamma_{i k}^{(2)} W_{k} \\
& +\bar{\kappa}\left(\left(\varepsilon_{1}+\varepsilon_{2}\right)\left(J_{3}\right)_{i}-6 \varepsilon_{1} \varepsilon_{2} \sum_{k=1}^{N_{x}} \Gamma_{i k}^{(2)}\left(J_{3}\right)_{k}\right) \\
& \left.\sum_{k=1}^{N_{x}} \Gamma_{i k}^{(3)} W_{k}+\left(-4 \varepsilon_{1} \varepsilon_{2} \bar{\kappa} \sum_{k=1}^{N_{x}} \Gamma_{i k}^{(1)}\left(J_{3}\right)_{k}\right) \sum_{k=1}^{N_{x}} \Gamma_{i k}^{(4)} W_{k}+\left(-\varepsilon_{1} \varepsilon_{2} \bar{\kappa}\left(J_{3}\right)_{i}\right) \sum_{k=1}^{N_{x}} \Gamma_{i k}^{(5)} W_{k}\right\}=0
\end{aligned}
$$$$
\left(-\varepsilon_{2} \alpha_{11}-2 \varepsilon_{1} \bar{\alpha}_{11}\right)_{i} \sum_{k=1}^{N_{x}} \Gamma_{i k}^{(4)} U_{k}+\left(\sum_{k=1}^{N_{x}} \Gamma_{i k}^{(1)}\left(-2 \varepsilon_{2} \alpha_{11}-4 \varepsilon_{1} \bar{\alpha}_{11}\right)_{k}\right) \sum_{k=1}^{N_{x}} \Gamma_{i k}^{(3)} U_{k}+
$$$$
\left(\left(\alpha_{11}+2 \bar{\alpha}_{11}\right)_{i}+\sum_{k=1}^{N_{x}} \Gamma_{i k}^{(2)}\left(-\varepsilon_{2} \alpha_{11}-2 \varepsilon_{1} \bar{\alpha}_{11}\right)_{k}\right) \sum_{k=1}^{N_{x}} \Gamma_{i k}^{(2)} U_{k}+\left(-\varepsilon_{2} \zeta_{11}-2 \varepsilon_{1} \bar{\zeta}_{11}\right)_{i} \sum_{k=1}^{N_{x}} \Gamma_{i k}^{(4)} \Psi_{k}+
$$$$
\left(\sum_{k=1}^{N_{x}} \Gamma_{i k}^{(1)}\left(-2 \varepsilon_{2} \zeta_{11}-4 \varepsilon_{1} \bar{\zeta}_{11}\right)_{k}\right) \sum_{k=1}^{N_{x}} \Gamma_{i k}^{(3)} \Psi_{k}
$$$$
+\left(\left(\zeta_{11}+2 \bar{\zeta}_{11}\right)_{i}+\sum_{k=1}^{N_{x}} \Gamma_{i k}^{(2)}\left(-\varepsilon_{2} \zeta_{11}-2 \varepsilon_{1} \bar{\zeta}_{11}\right)_{k}\right) \sum_{k=1}^{N_{x}} \Gamma_{i k}^{(2)} \Psi_{k}+
$$$$
+\left(4 \varepsilon_{1} \sum_{k=1}^{N_{x}} \Gamma_{i k}^{(1)} \bar{A}_{k}\right) \sum_{k=1}^{N_{x}} \Gamma_{i k}^{(1)} \Psi_{k}+\left(-2(\bar{A})_{i}+2 \varepsilon_{1} \sum_{k=1}^{N_{x}} \Gamma_{i k}^{(2)} \bar{A}_{k}\right) \Psi_{i}+
$$$$
\bar{\kappa}\left(-\varepsilon_{2} \delta_{11}-2 \varepsilon_{1} \bar{\delta}_{11}\right)_{i} \sum_{k=1}^{N_{x}} \Gamma_{i k}^{(5)} W_{k}+\bar{\kappa}\left(\sum_{k=1}^{N_{x}} \Gamma_{i k}^{(1)}\left(-2 \varepsilon_{2} \delta_{11}-4 \varepsilon_{1} \bar{\delta}_{11}\right)_{k}\right) \sum_{k=1}^{N_{x}} \Gamma_{i k}^{(4)} W_{k}+
$$ 


$$
\begin{aligned}
& \bar{\kappa}\left(\left(\delta_{11}+2 \bar{\delta}_{11}\right)_{i}+\sum_{k=1}^{N_{x}} \Gamma_{i k}^{(2)}\left(-\varepsilon_{2} \delta_{11}-2 \varepsilon_{1} \bar{\delta}_{11}\right)_{k}+2 \varepsilon_{1}(\bar{A})_{i}\right) \sum_{k=1}^{N_{x}} \Gamma_{i k}^{(3)} W_{k} \\
& +\left(4 \varepsilon_{1} \sum_{k=1}^{N_{x}} \Gamma_{i k}^{(1)} \bar{A}_{k}\right) \sum_{k=1}^{N_{x}} \Gamma_{i k}^{(2)} W_{k}+ \\
& \left(-2(\bar{A})_{i}+2 \varepsilon_{1} \sum_{k=1}^{N_{x}} \Gamma_{i k}^{(2)} \bar{A}_{k}\right) \sum_{k=1}^{N_{x}} \Gamma_{i k}^{(1)} W_{k}+ \\
& \omega^{2}\left\{\left(\left(-\hat{J}_{1}\right)_{i}+\left(\varepsilon_{1}+\varepsilon_{2}\right) \sum_{k=1}^{N_{x}} \Gamma_{i k}^{(2)}\left(\hat{J}_{1}\right)_{k}-\varepsilon_{1} \varepsilon_{2} \sum_{k=1}^{N_{x}} \Gamma_{i k}^{(4)}\left(\hat{J}_{1}\right)_{k}\right) U_{i}+\right. \\
& \left(2\left(\varepsilon_{1}+\varepsilon_{2}\right) \sum_{k=1}^{N_{x}} \Gamma_{i k}^{(1)}\left(\hat{J}_{1}\right)_{k}-4 \varepsilon_{1} \varepsilon_{2} \sum_{k=1}^{N_{x}} \Gamma_{i k}^{(3)}\left(\hat{J}_{1}\right)_{k}\right) \sum_{k=1}^{N_{x}} \Gamma_{i k}^{(1)} U_{k} \\
& +\left(\left(\varepsilon_{1}+\varepsilon_{2}\right)\left(\hat{J}_{1}\right)_{i}-6 \varepsilon_{1} \varepsilon_{2} \sum_{k=1}^{N_{x}} \Gamma_{i k}^{(2)}\left(\hat{J}_{1}\right)_{k}\right) \sum_{k=1}^{N_{x}} \Gamma_{i k}^{(2)} U_{k} \\
& +\left(-4 \varepsilon_{1} \varepsilon_{2} \sum_{k=1}^{N_{x}} \Gamma_{i k}^{(1)}\left(\hat{J}_{1}\right)_{k}\right) \sum_{k=1}^{N_{x}} \Gamma_{i k}^{(3)} U_{k}+\left(-\varepsilon_{1} \varepsilon_{2}\left(\hat{J}_{1}\right)_{i}\right) \sum_{k=1}^{N_{x}} \Gamma_{i k}^{(4)} U_{k}+ \\
& \left(\left(-\bar{J}_{2}\right)_{i}+\left(\varepsilon_{1}+\varepsilon_{2}\right) \sum_{k=1}^{N_{x}} \Gamma_{i k}^{(2)}\left(\bar{J}_{2}\right)_{k}-\varepsilon_{1} \varepsilon_{2} \sum_{k=1}^{N_{x}} \Gamma_{i k}^{(4)}\left(\bar{J}_{2}\right)_{k}\right) \Psi_{k} \\
& +\left(2\left(\varepsilon_{1}+\varepsilon_{2}\right) \sum_{k=1}^{N_{x}} \Gamma_{i k}^{(1)}\left(\bar{J}_{2}\right)_{k}-4 \varepsilon_{1} \varepsilon_{2} \sum_{k=1}^{N_{x}} \Gamma_{i k}^{(3)}\left(\bar{J}_{2}\right)_{k}\right) \\
& \sum_{k=1}^{N_{x}} \Gamma_{i k}^{(1)} \Psi_{k}+\left(\left(\varepsilon_{1}+\varepsilon_{2}\right)\left(\bar{J}_{2}\right)_{i}-6 \varepsilon_{1} \varepsilon_{2} \sum_{k=1}^{N_{x}} \Gamma_{i k}^{(2)}\left(\bar{J}_{2}\right)_{k}\right) \sum_{k=1}^{N_{x}} \Gamma_{i k}^{(2)} \Psi_{k} \\
& +\left(-4 \varepsilon_{1} \varepsilon_{2} \sum_{k=1}^{N_{x}} \Gamma_{i k}^{(1)}\left(\bar{J}_{2}\right)_{k}\right) \sum_{k=1}^{N_{x}} \Gamma_{i k}^{(3)} \Psi_{k}+ \\
& \left(-\varepsilon_{1} \varepsilon_{2}\left(\bar{J}_{2}\right)_{i}\right) \sum_{k=1}^{N_{x}} \Gamma_{i k}^{(4)} \Psi_{k}+\bar{\kappa}\left(\left(-\hat{J}_{4}\right)_{i}+\left(\varepsilon_{1}+\varepsilon_{2}\right) \sum_{k=1}^{N_{x}} \Gamma_{i k}^{(2)}\left(\hat{J}_{4}\right)_{k}-\varepsilon_{1} \varepsilon_{2} \sum_{k=1}^{N_{x}} \Gamma_{i k}^{(4)}\left(\hat{J}_{4}\right)_{k}\right) \sum_{k=1}^{N_{x}} \Gamma_{i k}^{(1)} W_{k}+
\end{aligned}
$$




$$
\begin{aligned}
& \bar{\kappa}\left(2\left(\varepsilon_{1}+\varepsilon_{2}\right) \sum_{k=1}^{N_{x}} \Gamma_{i k}^{(1)}\left(\hat{J}_{4}\right)_{k}-4 \varepsilon_{1} \varepsilon_{2} \sum_{k=1}^{N_{x}} \Gamma_{i k}^{(3)}\left(\hat{J}_{4}\right)_{k}\right) \sum_{k=1}^{N_{x}} \Gamma_{i k}^{(2)} W_{k} \\
& +\bar{\kappa}\left(\left(\varepsilon_{1}+\varepsilon_{2}\right)\left(\hat{J}_{4}\right)_{i}-6 \varepsilon_{1} \varepsilon_{2} \sum_{k=1}^{N_{x}} \Gamma_{i k}^{(2)}\left(\hat{J}_{4}\right)_{k}\right) \\
& \left.\sum_{k=1}^{N_{\chi}} \Gamma_{i k}^{(3)} W_{k}+\left(-4 \varepsilon_{1} \varepsilon_{2} \bar{\kappa} \sum_{k=1}^{N_{x}} \Gamma_{i k}^{(1)}\left(\hat{J}_{4}\right)_{k}\right) \sum_{k=1}^{N_{x}} \Gamma_{i k}^{(4)} W_{k}+\left(-\varepsilon_{1} \varepsilon_{2} \bar{\kappa}\left(\hat{J}_{4}\right)_{i}\right) \sum_{k=1}^{N_{x}} \Gamma_{i k}^{(5)} W_{k}\right\}=0 \\
& \bar{\kappa}\left(\varepsilon_{2} E_{11}+2 \varepsilon_{1} \bar{E}_{11}\right)_{i} \sum_{k=1}^{N_{x}} \Gamma_{i k}^{(5)} U_{k}+\bar{\kappa}\left(\sum_{k=1}^{N_{x}} \Gamma_{i k}^{(1)}\left(2 \varepsilon_{2} E_{11}+4 \varepsilon_{1} \bar{E}_{11}\right)_{k}\right) \sum_{k=1}^{N_{x}} \Gamma_{i k}^{(4)} U_{k}- \\
& \bar{\kappa}\left(\left(E_{11}+2 \bar{E}_{11}\right)_{i}+\sum_{k=1}^{N_{x}} \Gamma_{i k}^{(2)}\left(-\varepsilon_{2} E_{11}-2 \varepsilon_{1} \bar{E}_{11}\right)_{k}\right) \sum_{k=1}^{N_{x}} \Gamma_{i k}^{(3)} U_{k}+\bar{\kappa}\left(\varepsilon_{2} \delta_{11}+2 \varepsilon_{1} \bar{\delta}_{11}\right)_{i} \sum_{k=1}^{N_{x}} \Gamma_{i k}^{(5)} \Psi_{k}+ \\
& \bar{\kappa}\left(\sum_{k=1}^{N_{x}} \Gamma_{i k}^{(1)}\left(2 \varepsilon_{2} \delta_{11}+4 \varepsilon_{1} \bar{\delta}_{11}\right)_{k}\right) \sum_{k=1}^{N_{x}} \Gamma_{i k}^{(4)} \Psi_{k} \\
& -\bar{\kappa}\left(\left(\delta_{11}+2 \bar{\delta}_{11}\right)_{i}+\sum_{k=1}^{N_{x}} \Gamma_{i k}^{(2)}\left(-\varepsilon_{2} \delta_{11}-2 \varepsilon_{1} \bar{\delta}_{11}\right)_{k}+2 \varepsilon_{1}(\bar{A})_{i}\right) \\
& \sum_{k=1}^{N_{x}} \Gamma_{i k}^{(3)} \Psi_{k}+\left(-4 \varepsilon_{1} \sum_{k=1}^{N_{x}} \Gamma_{i k}^{(1)} \bar{A}_{k}\right) \sum_{k=1}^{N_{x}} \Gamma_{i k}^{(2)} \Psi_{k}+\left(2(\bar{A})_{i}-2 \varepsilon_{1} \sum_{k=1}^{N_{x}} \Gamma_{i k}^{(2)} \bar{A}_{k}\right) \sum_{k=1}^{N_{x}} \Gamma_{i k}^{(1)} \Psi_{k}+ \\
& \left(\bar{\kappa}^{2}\left(\varepsilon_{2} H_{11}+2 \varepsilon_{1} \bar{H}_{11}\right)+\varepsilon_{1} \varepsilon_{2} T\right)_{i} \sum_{k=1}^{N_{x}} \Gamma_{i k}^{(6)} W_{k}+\left(\bar{\kappa}^{2} \sum_{k=1}^{N_{x}} \Gamma_{i k}^{(1)}\left(2 \varepsilon_{2} H_{11}+4 \varepsilon_{1} \bar{H}_{11}\right)_{k}\right. \\
& \left.+5 \varepsilon_{1} \varepsilon_{2} \sum_{k=1}^{N_{x}} \Gamma_{i k}^{(1)} T_{k}\right) \sum_{k=1}^{N_{x}} \Gamma_{i k}^{(5)} W_{k}+ \\
& \left(\bar{\kappa}^{2}\left(-\left(H_{11}+2 \bar{H}_{11}\right)_{i}+\sum_{k=1}^{N_{x}} \Gamma_{i k}^{(2)}\left(\varepsilon_{2} H_{11}+2 \varepsilon_{1} \bar{H}_{11}\right)_{k}-2 \varepsilon_{1}(\bar{A})_{i}\right)-\left(\varepsilon_{1}+\varepsilon_{2}\right)(T)_{i}\right. \\
& \left.+10 \varepsilon_{1} \varepsilon_{2} \sum_{k=1}^{N_{x}} \Gamma_{i k}^{(2)} T_{k}\right)
\end{aligned}
$$


$\sum_{k=1}^{N_{x}} \Gamma_{i k}^{(4)} W_{k}+\left(-4 \varepsilon_{1} \sum_{k=1}^{N_{x}} \Gamma_{i k}^{(1)} \bar{A}_{k}-3\left(\varepsilon_{1}+\varepsilon_{2}\right) \sum_{k=1}^{N_{x}} \Gamma_{i k}^{(1)} T_{k}\right) \sum_{k=1}^{N_{x}} \Gamma_{i k}^{(3)} W_{k}+$

$\left(2(\bar{A})_{i}-2 \varepsilon_{1} \sum_{k=1}^{N_{x}} \Gamma_{i k}^{(2)} \bar{A}_{k}-3\left(\varepsilon_{1}+\varepsilon_{2}\right) \sum_{k=1}^{N_{x}} \Gamma_{i k}^{(2)} T_{k}+(T)_{i}\right) \sum_{k=1}^{N_{x}} \Gamma_{i k}^{(2)} W_{k}+\left(\sum_{k=1}^{N_{x}} \Gamma_{i k}^{(1)} T_{k}\right) \sum_{k=1}^{N_{x}} \Gamma_{i k}^{(1)} W_{k}+$

$\omega^{2}\left\{\bar{\kappa}\left(\left(J_{3}\right)_{i}-\left(\varepsilon_{1}+\varepsilon_{2}\right) \sum_{k=1}^{N_{x}} \Gamma_{i k}^{(2)}\left(J_{3}\right)_{k}+\varepsilon_{1} \varepsilon_{2} \sum_{k=1}^{N_{x}} \Gamma_{i k}^{(4)}\left(J_{3}\right)_{k}\right) \sum_{k=1}^{N_{x}} \Gamma_{i k}^{(1)} U_{k}+\right.$

$\bar{\kappa}\left(-2\left(\varepsilon_{1}+\varepsilon_{2}\right) \sum_{k=1}^{N_{x}} \Gamma_{i k}^{(1)}\left(J_{3}\right)_{k}+4 \varepsilon_{1} \varepsilon_{2} \sum_{k=1}^{N_{x}} \Gamma_{i k}^{(3)}\left(J_{3}\right)_{k}\right) \sum_{k=1}^{N_{x}} \Gamma_{i k}^{(2)} U_{k}$

$+\bar{\kappa}\left(-\left(\varepsilon_{1}+\varepsilon_{2}\right)\left(J_{3}\right)_{i}+6 \varepsilon_{1} \varepsilon_{2} \sum_{k=1}^{N_{x}} \Gamma_{i k}^{(2)}\left(J_{3}\right)_{k}\right)$

$\sum_{k=1}^{N_{x}} \Gamma_{i k}^{(3)} U_{k}+\left(4 \varepsilon_{1} \varepsilon_{2} \bar{\kappa} \sum_{k=1}^{N_{x}} \Gamma_{i k}^{(1)}\left(J_{3}\right)_{k}\right) \sum_{k=1}^{N_{x}} \Gamma_{i k}^{(4)} U_{k}+\left(\varepsilon_{1} \varepsilon_{2} \bar{\kappa}\left(J_{3}\right)_{i}\right) \sum_{k=1}^{N_{x}} \Gamma_{i k}^{(5)} U_{k}+$

$\bar{\kappa}\left(\left(\hat{J}_{4}\right)_{i}-\left(\varepsilon_{1}+\varepsilon_{2}\right) \sum_{k=1}^{N_{x}} \Gamma_{i k}^{(2)}\left(\hat{J}_{4}\right)_{k}+\varepsilon_{1} \varepsilon_{2} \sum_{k=1}^{N_{x}} \Gamma_{i k}^{(4)}\left(\hat{J}_{4}\right)_{k}\right) \sum_{k=1}^{N_{x}} \Gamma_{i k}^{(1)} \Psi_{k}+$

$\bar{\kappa}\left(-2\left(\varepsilon_{1}+\varepsilon_{2}\right) \sum_{k=1}^{N_{x}} \Gamma_{i k}^{(1)}\left(\hat{J}_{4}\right)_{k}+4 \varepsilon_{1} \varepsilon_{2} \sum_{k=1}^{N_{x}} \Gamma_{i k}^{(3)}\left(\hat{U}_{4}\right)_{k}\right) \sum_{k=1}^{N_{x}} \Gamma_{i k}^{(2)} \Psi_{k}$

$+\bar{\kappa}\left(-\left(\varepsilon_{1}+\varepsilon_{2}\right)\left(\hat{J}_{4}\right)_{i}+6 \varepsilon_{1} \varepsilon_{2} \sum_{k=1}^{N_{x}} \Gamma_{i k}^{(2)}\left(\hat{J}_{4}\right)_{k}\right)+$

$\sum_{k=1}^{N_{x}} \Gamma_{i k}^{(3)} \Psi_{k}+\left(4 \varepsilon_{1} \varepsilon_{2} \bar{\kappa} \sum_{k=1}^{N_{x}} \Gamma_{i k}^{(1)}\left(\hat{J}_{4}\right)_{k}\right) \sum_{k=1}^{N_{x}} \Gamma_{i k}^{(4)} \Psi_{k}+\left(\varepsilon_{1} \varepsilon_{2} \bar{\kappa}\left(\hat{J}_{4}\right)_{i}\right) \sum_{k=1}^{N_{x}} \Gamma_{i k}^{(5)} \Psi_{k}+$

$\left(\left(-J_{0}\right)_{i}+\left(\varepsilon_{1}+\varepsilon_{2}\right) \sum_{k=1}^{N_{x}} \Gamma_{i k}^{(2)}\left(J_{0}\right)_{k}+\varepsilon_{1} \varepsilon_{2} \sum_{k=1}^{N_{x}} \Gamma_{i k}^{(4)}\left(J_{0}\right)_{k}\right) W_{i}$

$+\left(2\left(\varepsilon_{1}+\varepsilon_{2}\right) \sum_{k=1}^{N_{x}} \Gamma_{i k}^{(1)}\left(J_{0}\right)_{k}+4 \varepsilon_{1} \varepsilon_{2} \sum_{k=1}^{N_{x}} \Gamma_{i k}^{(3)}\left(J_{0}\right)_{k}\right)$ 


$$
\begin{aligned}
& \sum_{k=1}^{N_{x}} \Gamma_{i k}^{(1)} W_{k}+\left(\left(\bar{\kappa}^{2} J_{6}+\left(\varepsilon_{1}+\varepsilon_{2}\right) J_{0}\right)_{i}-\bar{\kappa}^{2}\left(\varepsilon_{1}+\varepsilon_{2}\right) \sum_{k=1}^{N_{x}} \Gamma_{i k}^{(2)}\left(J_{6}\right)_{k}-6 \varepsilon_{1} \varepsilon_{2} \sum_{k=1}^{N_{x}} \Gamma_{i k}^{(2)}\left(J_{0}\right)_{k}\right. \\
& \left.+\bar{\kappa}^{2} \varepsilon_{1} \varepsilon_{2} \sum_{k=1}^{N_{x}} \Gamma_{i k}^{(4)}\left(J_{6}\right)_{k}\right) \\
& \sum_{k=1}^{N_{x}} \Gamma_{i k}^{(2)} W_{k}+\left(2 \bar{\kappa}^{2}\left(\varepsilon_{1}+\varepsilon_{2}\right) \sum_{k=1}^{N_{x}} \Gamma_{i k}^{(1)}\left(J_{6}\right)_{k}+4 \bar{\kappa}^{2} \varepsilon_{1} \varepsilon_{2} \sum_{k=1}^{N_{x}} \Gamma_{i k}^{(3)}\left(J_{6}\right)_{k}\right. \\
& \left.-4 \varepsilon_{1} \varepsilon_{2} \sum_{k=1}^{N_{x}} \Gamma_{i k}^{(1)}\left(J_{0}\right)_{k}\right) \sum_{k=1}^{N_{x}} \Gamma_{i k}^{(3)} W_{k}+ \\
& \left(\left(-\bar{\kappa}^{2}\left(\varepsilon_{1}+\varepsilon_{2}\right) J_{6}-\varepsilon_{1} \varepsilon_{2} J_{0}\right)_{i}+6 \bar{\kappa}^{2} \varepsilon_{1} \varepsilon_{2} \sum_{k=1}^{N_{x}} \Gamma_{i k}^{(2)}\left(J_{6}\right)_{k}\right) \sum_{k=1}^{N_{x}} \Gamma_{i k}^{(4)} W_{k} \\
& +\left(4 \bar{\kappa}^{2} \varepsilon_{1} \varepsilon_{2} \sum_{k=1}^{N_{x}} \Gamma_{i k}^{(1)}\left(J_{6}\right)_{k}\right) \sum_{k=1}^{N_{x}} \Gamma_{i k}^{(5)} W_{k}+ \\
& \left.\left(\bar{\kappa}^{2} \varepsilon_{1} \varepsilon_{2}\left(J_{6}\right)_{i}\right) \sum_{k=1}^{N_{x}} \Gamma_{i k}^{(6)} W_{k}\right\}=0
\end{aligned}
$$

where $U_{i}=U\left(x_{i}\right), W_{i}=W\left(x_{i}\right)$ and $\Psi_{i}=\Psi\left(x_{i}\right)$.

Equations (39) and (41) can be rewritten as:

$$
\left[\mathbf{K}-\omega^{2} \mathbf{M}\right] \overline{\mathbf{W}}=\mathbf{0}
$$

where

$$
\overline{\mathbf{W}}=\left\{\begin{array}{c}
\mathbf{U} \\
\mathbf{\Psi} \\
\mathbf{W}
\end{array}\right\}
$$

and the $i^{\text {th }}$ element of the vectors $\mathbf{U}, \mathbf{W}$ and $\Psi$ are $U_{i}, W_{i}$ and $\Psi_{i}$, respectively. Also, $\mathbf{M}$ and $\mathbf{K}$ are the equivalent mass and stiffness matrices obtained by properly arranging the elements from Eqs. (39) to (41). 
For eigenvalue analysis, Eq. (42) and the boundary conditions should be satisfied simultaneously. Based on GDQM, the boundary conditions can be written as constraints on the degrees of freedom as:

$\mathrm{R}_{1}^{\mathrm{T}} \overline{\mathbf{W}}=\mathbf{0}$

where $\mathbf{R}_{\mathbf{1}}$ is matrix that depends on the type of boundary conditions. Boundary conditions involving derivatives can be defined in the form of Eq. (44) by using Eq. (34). For example, the clamped-clamped boundary conditions are given by:

$U_{1}=\Psi_{1}=W_{1}=U_{N_{x}}=\Psi_{N_{x}}=W_{N_{x}}=0$

$U_{1}^{\prime}=\Psi_{1}^{\prime}=W_{1}^{\prime}=U_{N_{x}}^{\prime}=\Psi_{N_{x}}^{\prime}=W_{N_{x}}^{\prime}=0$

The boundary conditions may be rewritten using the DQM approximation as follows:

for $i=1(x=0) \Rightarrow\left\{\begin{array}{c}U_{1}=W_{1}=\Psi_{1}=0 \\ \left.\frac{\partial U}{\partial x}\right|_{i=1}=\sum_{j=1}^{N_{x}} \Gamma_{1 j}^{(1)} U_{j}=0 \\ \left.\frac{\partial W}{\partial x}\right|_{i=1}=\sum_{j=1}^{N_{x}} \Gamma_{1 j}^{(1)} W_{j}=0 \\ \left.\frac{\partial \Psi}{\partial x}\right|_{i=1}=\sum_{j=1}^{N_{x}} \Gamma_{1 j}^{(1)} \Psi_{j}=0\end{array}\right.$

for $i=N_{x}(x=L) \Rightarrow\left\{\begin{array}{c}U_{N_{x}}=W_{N_{x}}=\Psi_{N_{x}}=0 \\ \left.\frac{\partial U}{\partial x}\right|_{i=N_{x}}=\sum_{j=1}^{N_{x}} \Gamma_{N_{x} j}^{(1)} U_{j}=0 \\ \left.\frac{\partial W}{\partial x}\right|_{i=N_{x}}=\sum_{j=1}^{N_{x}} \Gamma_{N_{x} j}^{(1)} W_{j}=0 \\ \left.\frac{\partial \Psi}{\partial x}\right|_{i=N_{x}}=\sum_{j=1}^{N_{x}} \Gamma_{N_{x} j}^{(1)} \Psi_{j}=0\end{array}\right.$

These constraint equations are all linear expressions in $U_{j}, \Psi_{j}$ and $W_{j}$, and hence $\mathbf{R}_{\mathbf{1}}$ in Eq. (44) is readily determined. For other boundary conditions, such as simply supported or free, we can easily determine the matrix $\mathbf{R}_{\mathbf{1}}$ using a similar approach.

where $\mathbf{R}_{b}$ is square and non-singular. A suitable transformation is then 


$$
\mathbf{T}_{\mathbf{1}}=\left\{\begin{array}{c}
-\mathbf{R}_{b}^{-1} \mathbf{R}_{d} \\
\mathbf{I}
\end{array}\right\}
$$

which eliminates the boundary degrees of freedom since

$$
\overline{\mathbf{W}}=\left\{\begin{array}{l}
\overline{\mathbf{W}}_{b} \\
\overline{\mathbf{W}}_{d}
\end{array}\right\}=\mathbf{T}_{\mathbf{1}} \overline{\mathbf{W}}_{d}
$$

The mass and stiffness matrices are then rearranged to match the ordering given in Eq. (47), and the transformed mass and stiffness matrices are

$$
\overline{\mathbf{M}}=\mathbf{T}_{\mathbf{1}}^{\mathbf{T}} \mathbf{M T}_{\mathbf{1}} \text { and } \overline{\mathbf{K}}=\mathbf{T}_{\mathbf{1}}^{\mathbf{T}} \mathbf{K T}_{\mathbf{1}}
$$

The eigenvalue problem then becomes

$$
\left[\overline{\mathbf{K}}-\omega^{2} \overline{\mathbf{M}}\right] \overline{\mathbf{W}}_{d}=\mathbf{0}
$$

\section{Numerical Results and Discussion}

The numerical results for the vibration analysis of a 2D-FG rotating nanobeam with CC boundary conditions are now computed and discussed by varying five parameters: $n x, n z, \gamma, \Omega, L / h$. Thus, the effects of the power law indexes, $n x, n z$, the porosity volume fraction, $\gamma$, the dimensionless beam rotating velocity, $\Omega$, and the ratio of length to thickness of the nanobeam, $L / h$, are shown. For each parameter, the remaining four parameters are fixed to their baseline values and the parameter of interest is then varied. The effects of clamped-simply supported (CS) and simply supported (SS) are also considered. The material properties of the 2D nanobeam are given in Table 1.

Table 1: Material Properties [4]

\begin{tabular}{ccccccc}
\hline Material & $\boldsymbol{\lambda}(\boldsymbol{G P a})$ & $\boldsymbol{\mu}(\boldsymbol{G P a})$ & $\boldsymbol{\varepsilon}_{\mathbf{1}}\left(\boldsymbol{n m}^{\mathbf{2}}\right)$ & $\boldsymbol{\varepsilon}_{\mathbf{2}}\left(\boldsymbol{n m}^{\mathbf{2}}\right)$ & $\boldsymbol{a}(\boldsymbol{n m})$ & $\boldsymbol{\rho}\left(\boldsymbol{k g} / \boldsymbol{m}^{\mathbf{3}}\right)$ \\
\hline Pure Metal $(\mathrm{Cu})$ & 25 & 78 & $0.17 a^{2}$ & $0.03 a^{2}$ & 0.3597 & 8960 \\
Pure Ceramic (Bao) & 48.1 & 38.8 & $0.15 a^{2}$ & $0.045 a^{2}$ & 0.5537 & 5720 \\
\hline
\end{tabular}

\subsection{Model Validation}

To validate the present model and for verification of the accuracy of the results, the numerical results from the present model are compared to the numerical results in Ref. [25]. For this purpose, we reduced our proposed beam model to the Eringen / Timoshenko beam model by 
assuming $\sqrt{\varepsilon_{1}}=\sqrt{\varepsilon_{2}}=\mu$ and $\bar{\kappa}=0$ which was used in Ref. [25]. In addition, the results in Table 2 are shown for the Eringen / Reddy beam model by considering $\bar{\kappa}=\frac{-4}{3 h^{2}} \neq 0$. The comparison of the results in Table 2 shows good agreement based for the Eringen / Timoshenko beam model and exact agreement for the Eringen / Reddy beam model, which demonstrates the accuracy of the proposed model.

Table 2: Model validation by comparison to the results of Ref. [25] for the FGM nanobeam

\begin{tabular}{|c|c|c|c|c|c|c|c|c|}
\hline & & & \multirow{2}{*}{\multicolumn{3}{|c|}{$\Omega=0(\mathrm{Grad} / \mathrm{s})$}} & \multirow{2}{*}{\multicolumn{3}{|c|}{$\gamma=0.1$}} \\
\hline & & & & & & & & \\
\hline & & & $n x=0$ & $n x=1$ & $n x=5$ & $n x=0$ & $n x=1$ & $n x=5$ \\
\hline \multirow{9}{*}{$\varepsilon_{1}=\varepsilon_{2}=0$} & \multirow{3}{*}{$n z=0$} & Present $\bar{\kappa}=0$ & 21.2375 & 15.0436 & 12.4832 & 22.3012 & 15.0738 & 12.2033 \\
\hline & & Present $\bar{\kappa} \neq 0$ & 21.3846 & 15.2570 & 12.5657 & 22.3200 & 15.2128 & 12.2875 \\
\hline & & Ref. [25] & 21.3846 & 15.2570 & 12.5657 & 22.3200 & 15.2128 & 12.2875 \\
\hline & \multirow{3}{*}{$n z=1$} & Present $\bar{\kappa}=0$ & 15.0735 & 13.2223 & 12.1179 & 15.2801 & 12.9821 & 11.7324 \\
\hline & & Present $\bar{\kappa} \neq 0$ & 15.3286 & 13.3248 & 12.1382 & 15.3013 & 13.1017 & 11.8263 \\
\hline & & Ref. [25] & 15.3286 & 13.3248 & 12.1382 & 15.3013 & 13.1017 & 11.8263 \\
\hline & \multirow{3}{*}{$n z=5$} & Present $\bar{\kappa}=0$ & 13.0101 & 12.3084 & 11.8532 & 12.7321 & 12.0001 & 11.5073 \\
\hline & & Present $\bar{\kappa} \neq 0$ & 13.0963 & 12.3806 & 11.9239 & 12.8608 & 12.0864 & 11.5955 \\
\hline & & Ref. [25] & 13.0963 & 12.3806 & 11.9239 & 12.8608 & 12.0864 & 11.5955 \\
\hline \multirow{9}{*}{$\begin{array}{l}\sqrt{\varepsilon_{1}}=\sqrt{\varepsilon_{2}} \\
=0.15\end{array}$} & \multirow{3}{*}{$n z=0$} & Present $\bar{\kappa}=0$ & 18.6636 & 13.2903 & 11.0601 & 19.5762 & 13.3258 & 10.7923 \\
\hline & & Present $\bar{\kappa} \neq 0$ & 18.9388 & 13.4903 & 11.0739 & 19.7673 & 13.4461 & 10.8230 \\
\hline & & Ref. [25] & 18.9388 & 13.4903 & 11.0739 & 19.7673 & 13.4461 & 10.8230 \\
\hline & \multirow{3}{*}{$n z=1$} & Present $\bar{\kappa}=0$ & 13.5174 & 11.6252 & 10.6661 & 13.4925 & 11.5012 & 10.4321 \\
\hline & & Present $\bar{\kappa} \neq 0$ & 13.5734 & 11.7965 & 10.7242 & 13.548 & 11.5981 & 10.4459 \\
\hline & & Ref. [25] & 13.5734 & 11.7965 & 10.7242 & 13.548 & 11.5981 & 10.4459 \\
\hline & \multirow{3}{*}{$n z=5$} & Present $\bar{\kappa}=0$ & 11.5073 & 10.9523 & 10.4210 & 11.3273 & 10.6891 & 10.2499 \\
\hline & & Present $\bar{\kappa} \neq 0$ & 11.6006 & 10.9653 & 10.5499 & 11.3922 & 10.7048 & 10.2581 \\
\hline & & Ref. [25] & 11.6006 & 10.9653 & 10.5499 & 11.3922 & 10.7048 & 10.2581 \\
\hline
\end{tabular}




\subsection{The Effects of $n x$ and $\mathrm{nz}$}

The effects of the power law indexes, $n z$ and $n x$, on the natural frequency are illustrated in Fig. 2. The fundamental natural frequencies of perfect nanobeams are given for different values of power indexes $n z$ and $n x$. Clearly, due to the decrease in the stiffness of the nanobeam, increasing $n x$ and $n z$ decreases the natural frequency. Also, for larger $n z, n x$ (approximately more than 2) the frequency decreases much more slowly and converges to a constant value.

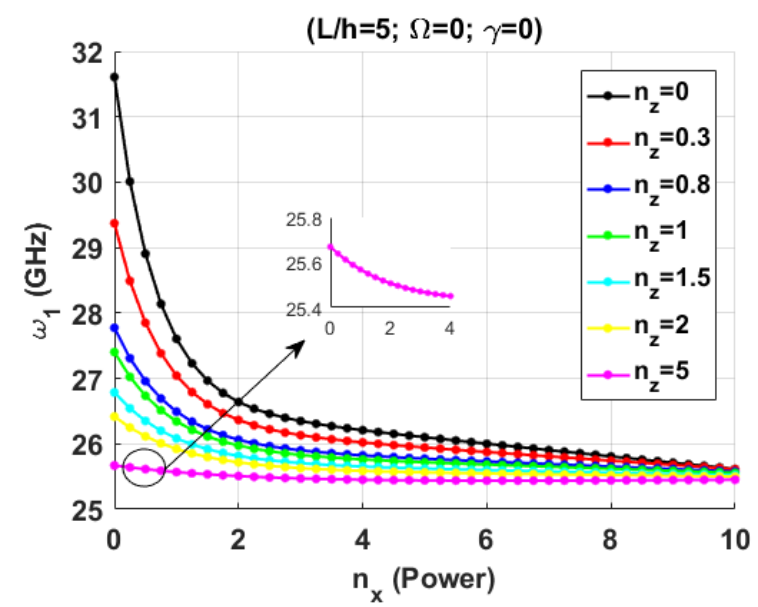

Fig 2. The effects of $n x$ and $n z$ on the fundamental natural frequency.

\subsection{The Effects of $\Omega$}

The effect of the nanobeam dimensionless rotating velocity $\Omega$ on the natural frequency is now considered. The dimensionless beam rotating velocity is defined as:

$$
\Omega=\widetilde{\omega} L^{2} \sqrt{\frac{\rho_{c} A}{E_{c} I}}
$$

where $A, \widetilde{\omega}, L, I$ are the cross-section area, the rotating velocity, the length, and the second moment of cross-section area for the nanobeam, respectively. Also, $\rho_{c}$ is the density and $E_{c}$ is the Young's modulus for pure ceramic in the FGM. The effects of $\Omega$, in combination with other values of parameters $n x, n z, \gamma, L / h$, are given in Figs. 3 to 7 .

Figure 3 shows the fundamental natural frequency as $\Omega$ varies for different values of $n x$, and for different values of $n z$, namely $n z=0, n z=0.5, n z=1.5$ and $n z=5$. Figure 3 shows that increasing $\Omega$ up to certain value, increases the fundamental natural frequency. However, after a certain value (which is named the corner rotating velocity, $\Omega_{c}$ ) increasing $\Omega$ has no significant 
effect on the natural frequency and it becomes constant. The value of the corner rotating velocity depends on $n z$ and $n x$; increasing $n z$ and $n x$ decreases the corner rotating velocity. However, the effect of $n_{x}$ on $\Omega_{c}$ is more significant than the effect of $n_{z}$.

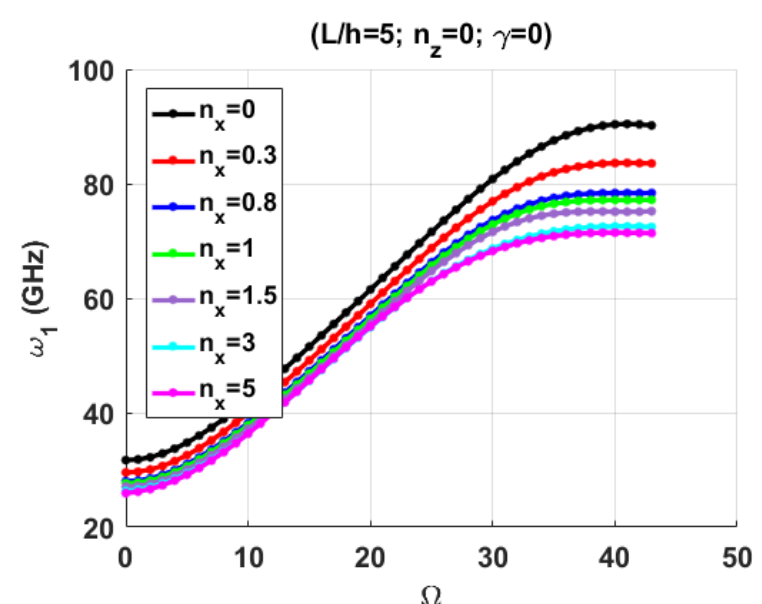

(a)

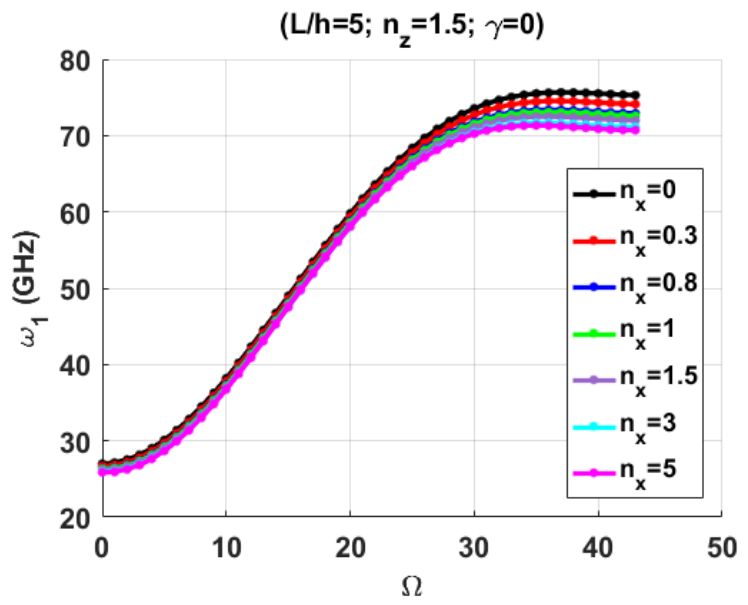

(c)

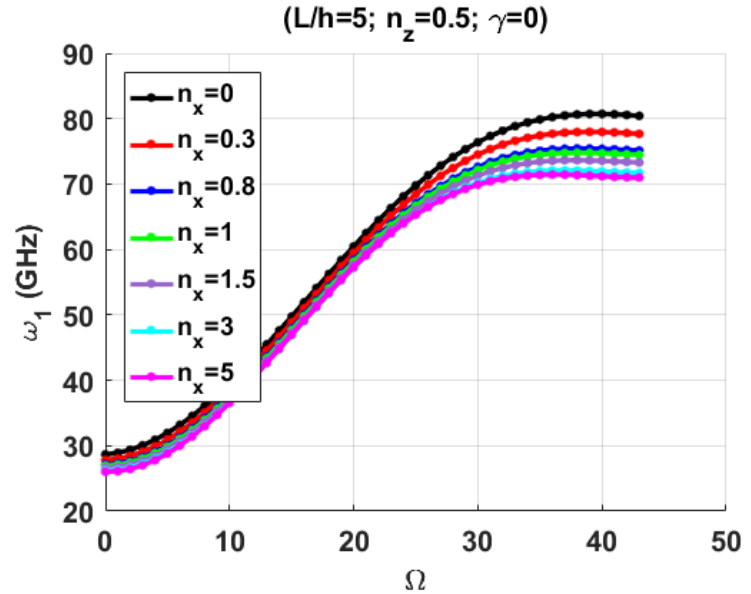

(b)

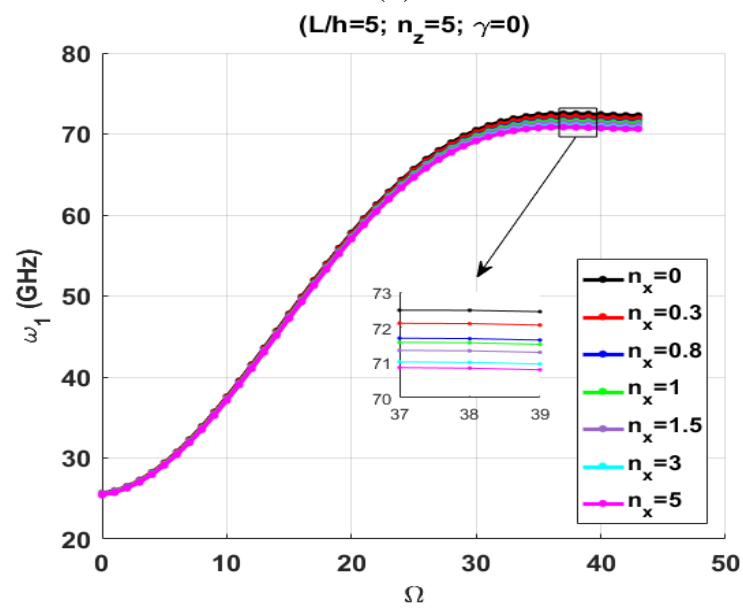

(d)

Fig 3. The effects of rotational velocity $(\Omega)$ on the fundamental natural frequency by varying $n x$ and $n z$.

(a) $n z=0$

(b) $n z=0.5$

(c) $n s=1.5$

(d) $n z=5$

Figure 4 is similar to Fig. 3 but the roles of $n x$ and $n z$ are interchanged. The results are observed for different values of $n x$, namely $n x=0, n x=0.5, n x=1.5$ and $n x=5$. Similarly to Fig. 3, increasing $\Omega$ up to $\Omega_{c}$, increases the value of the fundamental natural frequency, $\omega_{1}$, but for $\Omega>\Omega_{c}$ the effect on $\omega_{1}$ is only small. 


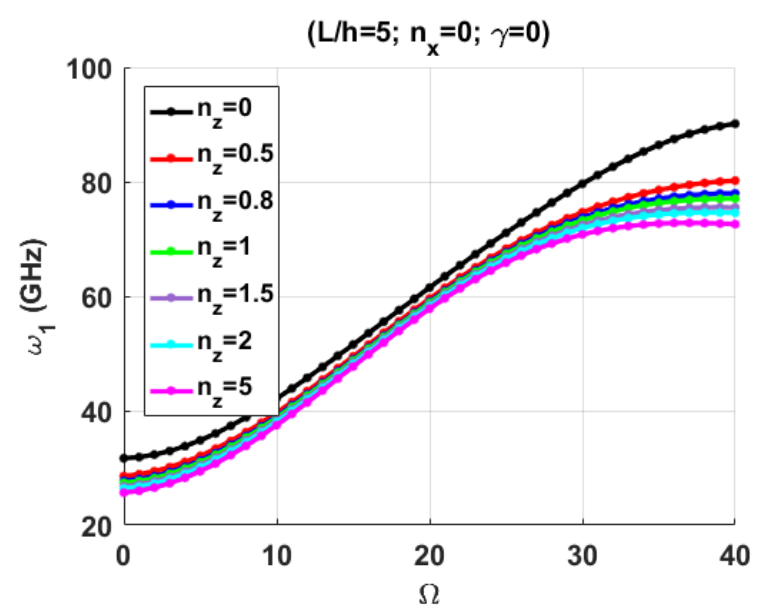

(a)

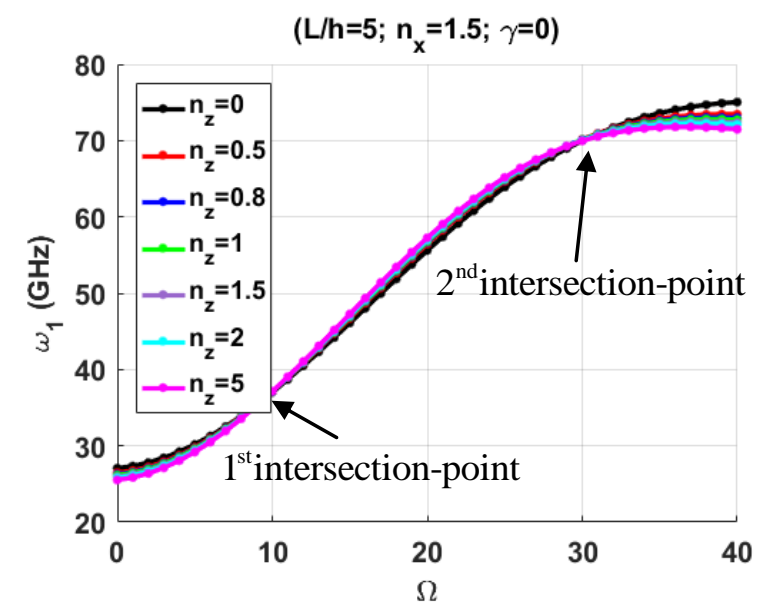

(c)

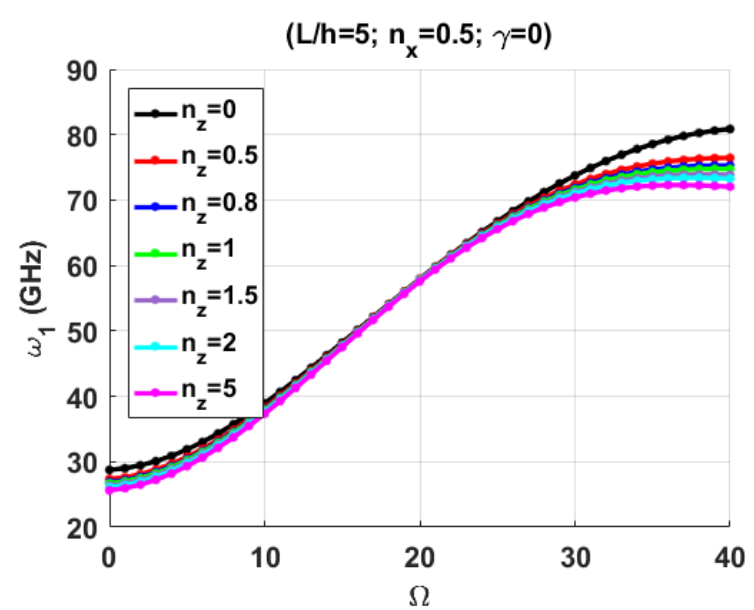

(b)

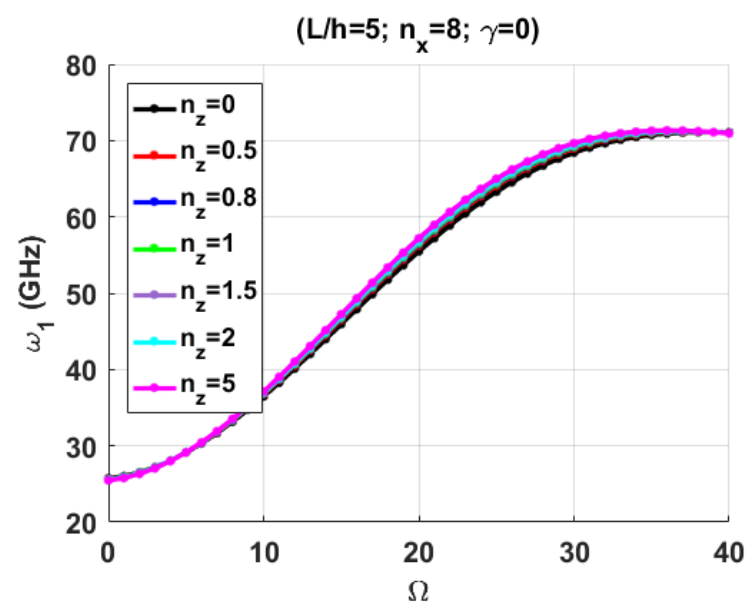

(d)

Fig 4. The effects of rotational velocity $(\Omega)$ on the fundamental natural frequency by varying $n x$ and $n z . \quad \begin{array}{llll}\text { (a) } n x=0 & \text { (b) } n x=0.5 & \text { (c) } n x=1.5 & \text { (d) } n x=8\end{array}$

In Fig. 4, with increasing $n x$, the plots get to be closer to each other. For $n x>0$, the diagrams consist of three zones and there are 2 intersection points which are named the point of convergence and the corner point, respectively. Before the point of convergence, increasing $n z$ causes the fundamental non-dimensional frequency to decrease. After the convergence point, the response of the fundamental non-dimensional frequency is reversed. The response also changes its behavior at the corner point. After the corner point, the variation of $n z$ has no significant effect on the fundamental non-dimensional frequency. The reason is that when $n z$ is low, the effect of the dimensionless beam rotating velocity is intense but as the nz increases, the effect of the rotating velocity dominates the effect of the power index. Thus, for low dimensionless beam rotating velocity, increasing $n z$ decreases the fundamental non-dimensional frequency. However 
when the dimensionless beam rotating velocity increases to above the point of convergence, $n z$ acts inversely.

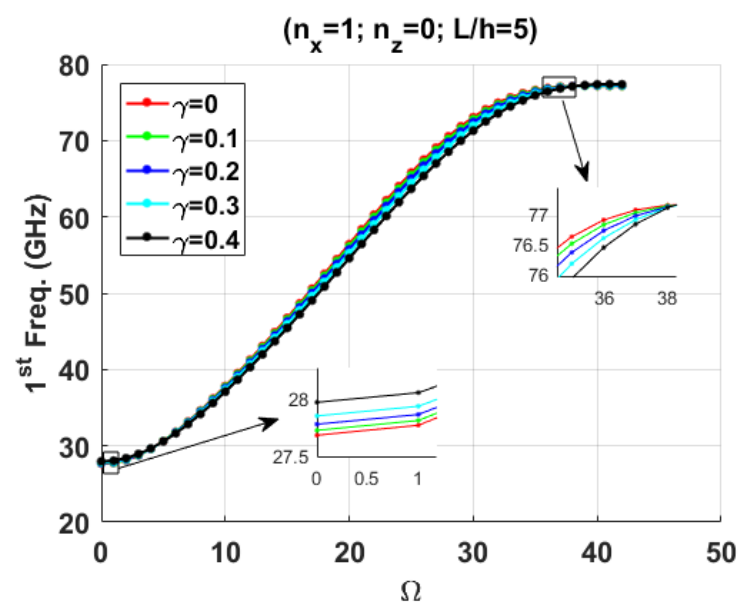

(a)

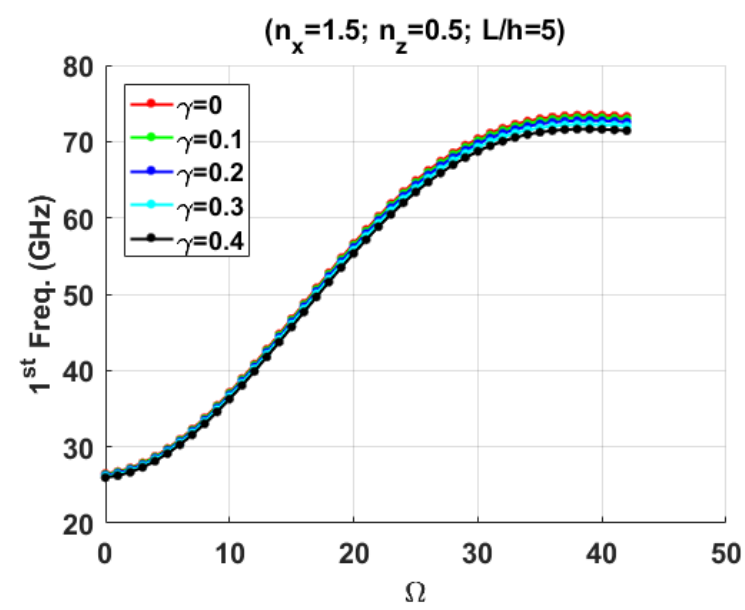

(b)

Fig 5 . The effects of rotational velocity $(\Omega)$ on the fundamental natural frequency by varying $\gamma . \square \square \square \square$ (a) $n x=1 ; n z=0 \quad$ (b) $n x=1.5 ; n z=0.5$

Figure 5 shows the effect of $\Omega$ and $\gamma$ on the fundamental natural frequency, and the trends are similar to those in Fig. 4. According to Fig. 5, the variation of $\gamma$ has no significant effect on the corner rotating velocity.

The effects of $\Omega$ on the fundamental natural frequency of the nanobeam due to the variation of the length to thickness ratio are shown in Fig. 6. The results are given for two different states, one for $n x=n z=0$ and the other for $n x=1.5$ and $n z=0.5$. Clearly, increasing the ratio of length to thickness, not only decreases the natural frequency in the range $\Omega<\Omega_{c}$, but it also increases the corner rotating velocity. Also, the maximum fundamental natural frequency for all values of $L / h$ is constant and is achieved at the corner rotating velocity. 


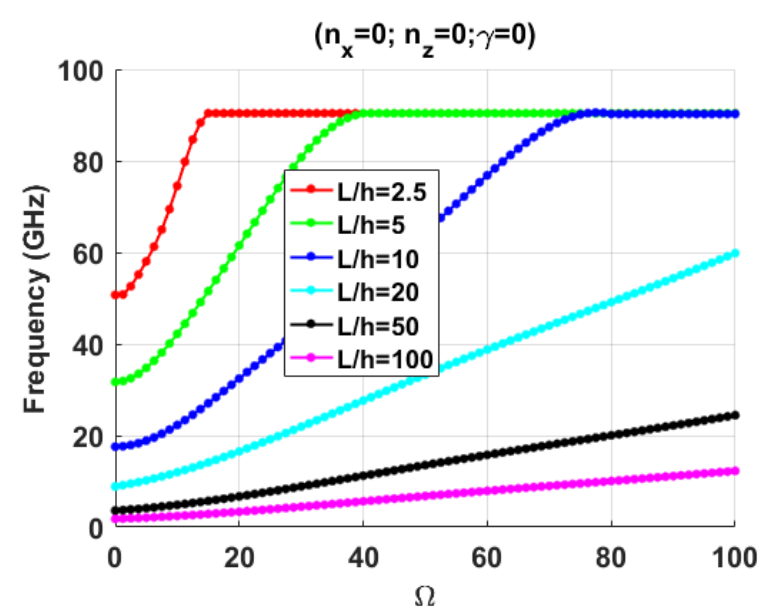

(a)

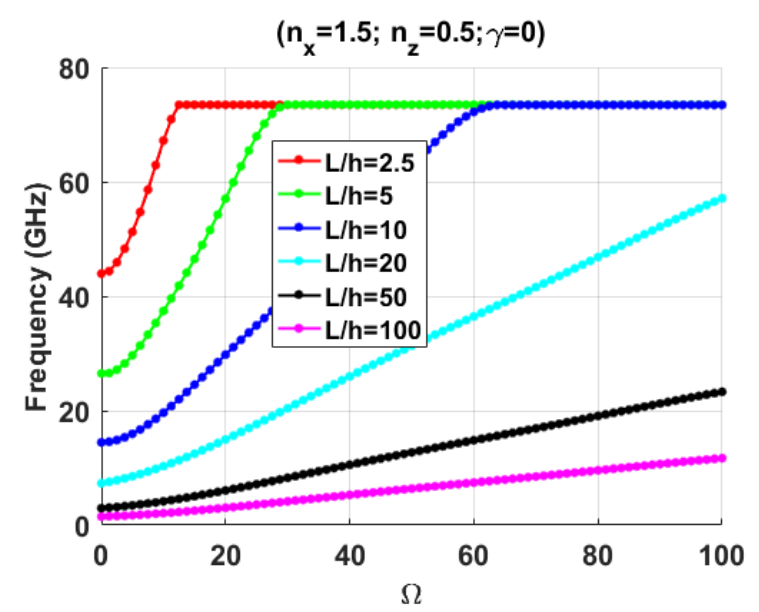

(b)

Fig 6. The effects of $\Omega$ on the fundamental natural frequency for different length to thickness ratios. $\quad \begin{array}{ll}\text { (a) } n x=n z=0 & \text { (b) } n x=1.5 ; n z=0.5\end{array}$

\subsection{The Effects of $\gamma$}

Figure 7 shows the fundamental natural frequency of the nanobeam versus the power index in the $x$ direction $(n x)$ by considering the variation of $\gamma$ from 0 to 0.4 and 3 different values for $n z$. Increasing $n x$ decreases the natural frequency $\omega_{1}$ for constant $\gamma$. In addition, the effect of $\gamma$ on $\omega_{1}$ depends on the values of $n z$ and $n x$. For $n z<1$, increasing $\gamma$ has different trends for the fundamental natural frequency depending on $n x$. Thus, for $n x$ below a specific value (here $n x \approx 2$ for $n z=0$ and $n x \approx 0.6$ for $n z=0.5$ ) the natural frequency increases with increasing $\gamma$. However, for larger $n x, \omega_{1}$ decreases. In contrast, for $n z>1$ the fundamental natural frequency decreases with increasing $\gamma$ for all values of $n x$. 


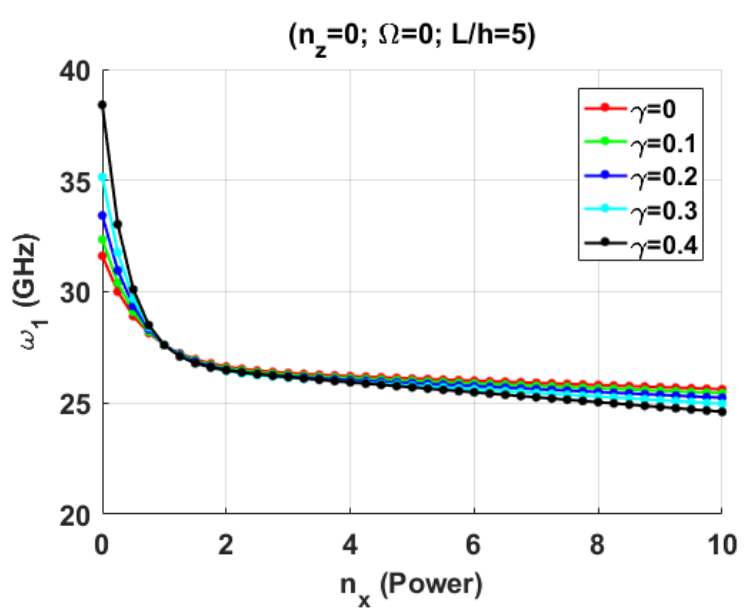

(a)

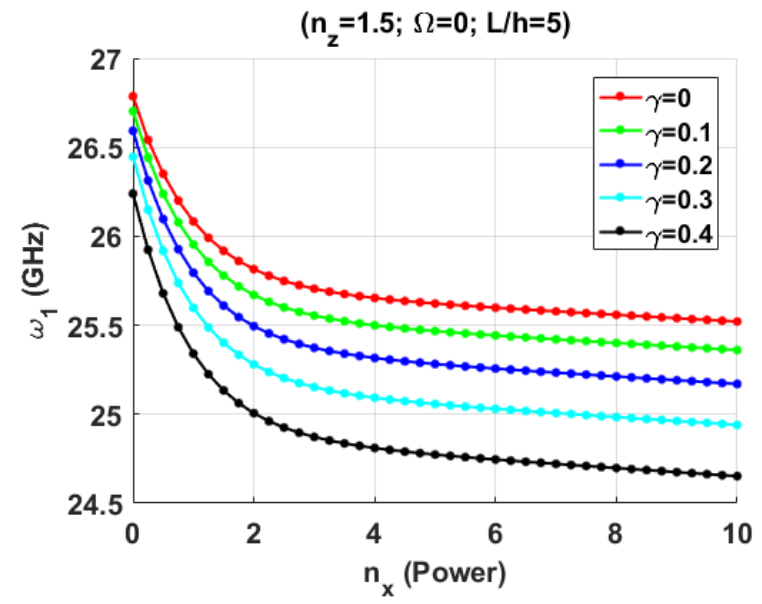

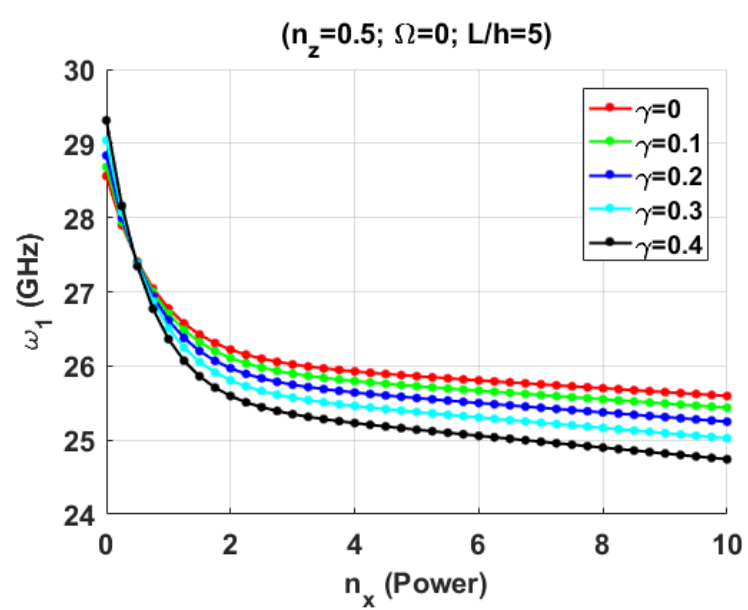

(b)

(c)

Fig 7. The effects of $\gamma$ on the fundamental natural frequency, as $n x$ varies.
(a) $n z=0$
(b) $n z=0.5$
(c) $n z=1.5$

\subsection{The Effects of $L / h$}

Figure 8 shows the effects of varying the length to thickness ratio $(L / h)$ on the fundamental natural frequency. The results are plotted for natural frequency versus $n x$ for different values of $L / h$ and $n z=0$ or 0.5 . Clearly, increasing $L / h$ causes the natural frequency to decrease. In addition, increasing $n z$ and/or $n x$ decreases the natural frequency for all values of $L / h$. 


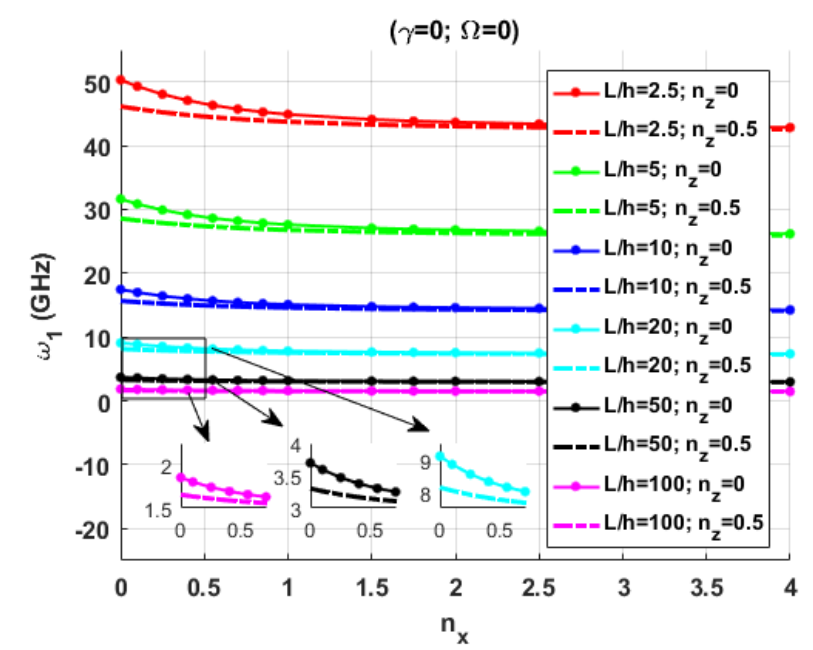

Fig 8 . The effects of $L / h$ on the fundamental natural frequency with varying $n x$

To show the comprehensiveness of the developed model, we extract the results for the Timoshenko beam model and compare them to the results for the Reddy beam model in Fig. 9. The results show that using the TBT model reduces the accuracy and is the main reason that many researchers [46-49] have employed higher order beam theory to model nanobeams. Here, we considered the higher order beam theory in order to study comprehensive and more accurate vibration analysis. 


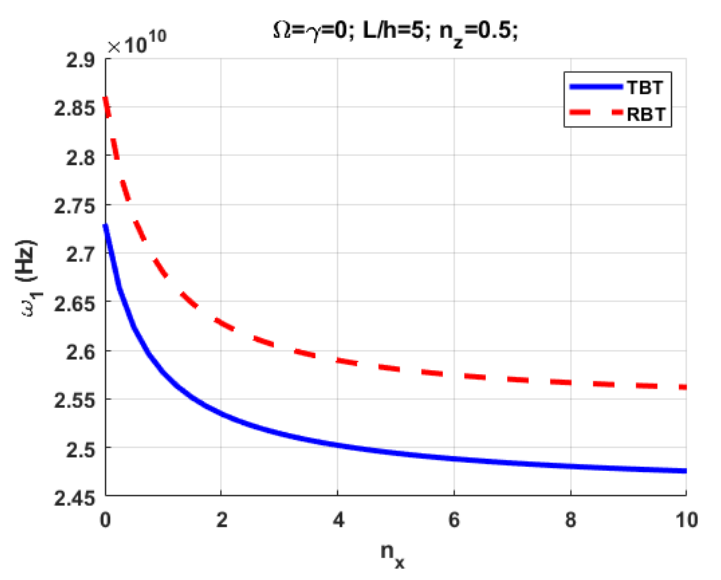

(a)

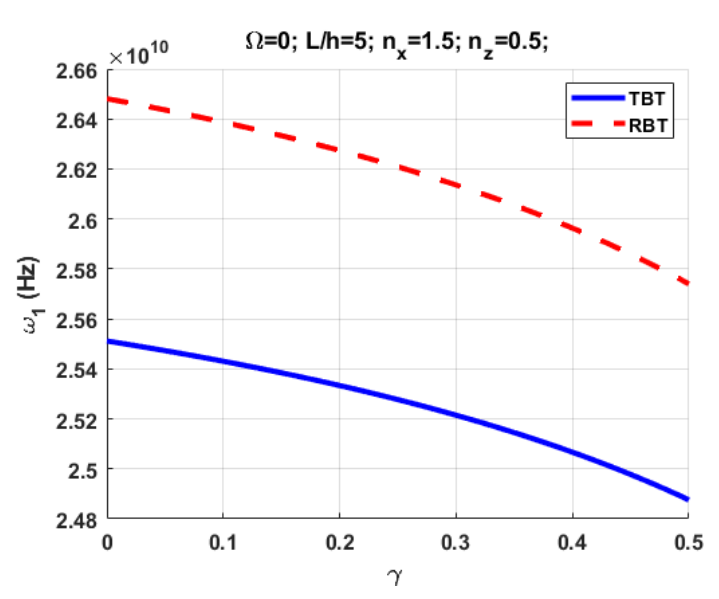

(c)

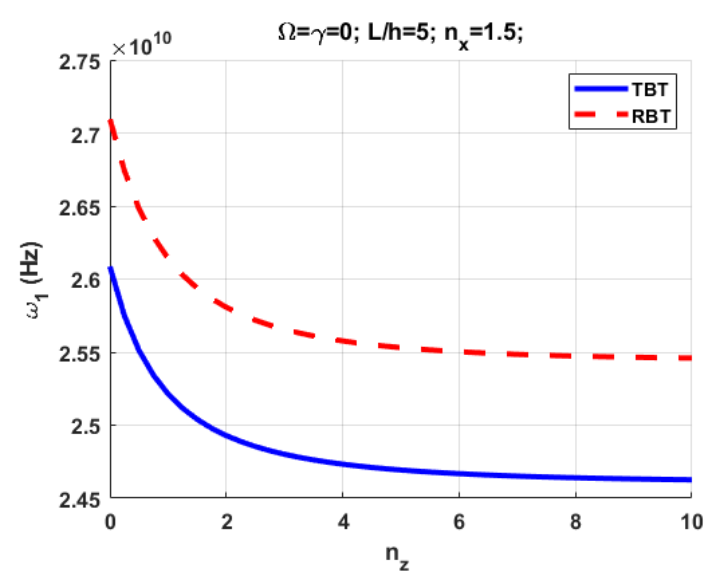

(b)

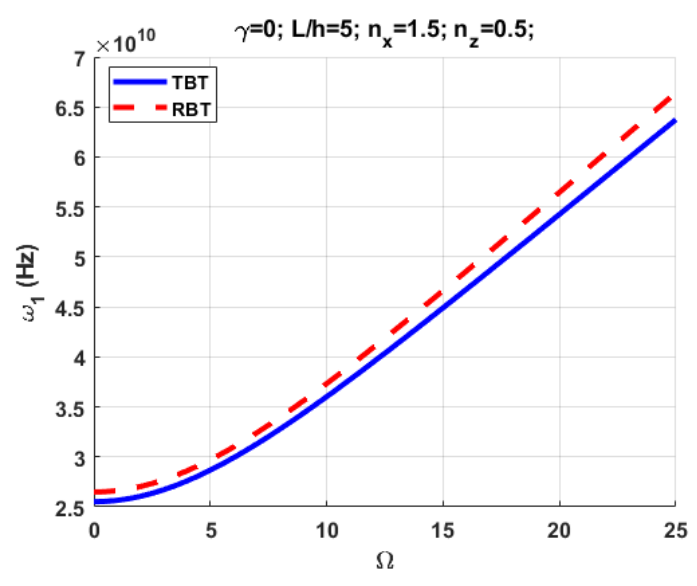

(d)

Fig 9. Comparison between Timoshenko (TBT) and Reddy (RBT) beam models for varying
(a) $n x$
(b) $n z$
(c) $\gamma$
(d) $\Omega$

\subsection{The Effect of the Nonlocal Parameters $\left(\epsilon_{1}, \epsilon_{2}\right)$}

Since, in a 2D-FG nano beam, the material configuration alters along both the longitudinal and the thickness directions, the constant nonlocal parameters $\epsilon_{1}$ and $\epsilon_{2}$ also alter along the longitudinal (x-axis) and thickness (z-axis) directions, similarly to the other mechanical properties, as follows:

$$
\begin{aligned}
& \epsilon_{1}(x, z)=\left(\epsilon_{1}\right)_{m}+\left[\left(\epsilon_{1}\right)_{c}-\left(\epsilon_{1}\right)_{m}\right]\left(\frac{1}{2}+\frac{z}{h}\right)^{n z}\left(\frac{x}{L}\right)^{n x}-\frac{\gamma}{2}\left[\left(\epsilon_{1}\right)_{c}+\left(\epsilon_{1}\right)_{m}\right] \\
& \epsilon_{2}(x, z)=\left(\epsilon_{2}\right)_{m}+\left[\left(\epsilon_{2}\right)_{c}-\left(\epsilon_{2}\right)_{m}\right]\left(\frac{1}{2}+\frac{z}{h}\right)^{n z}\left(\frac{x}{L}\right)^{n x}-\frac{\gamma}{2}\left[\left(\epsilon_{2}\right)_{c}+\left(\epsilon_{2}\right)_{m}\right]
\end{aligned}
$$


where the ()$_{m}$ and ()$_{c}$ subscripts denote the metal and ceramic properties respectively.

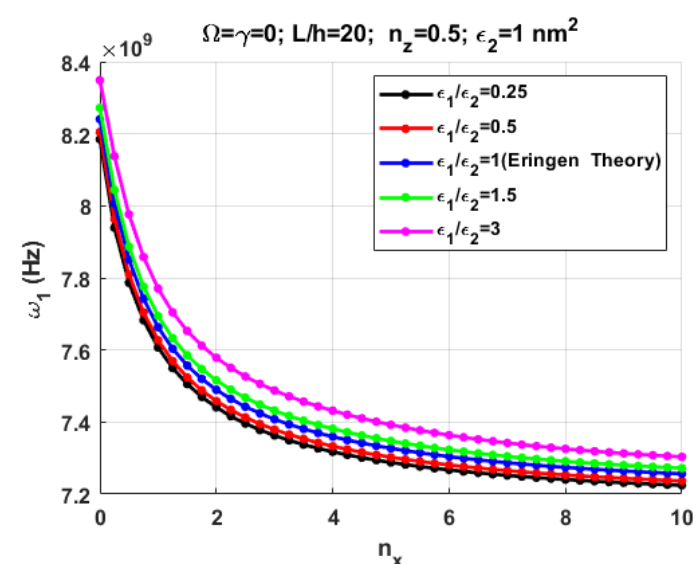

(a)

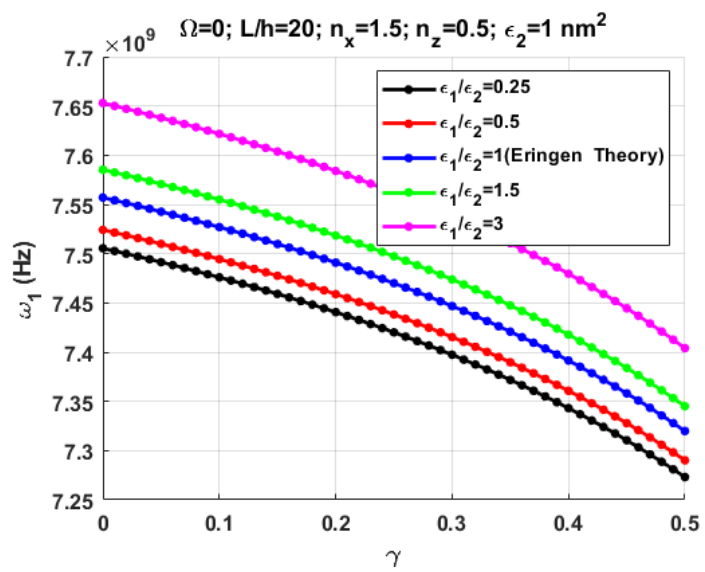

(c)

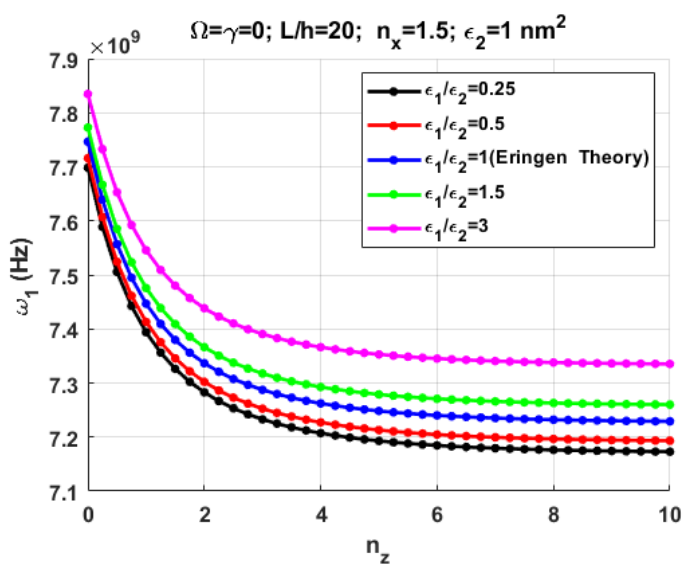

(b)

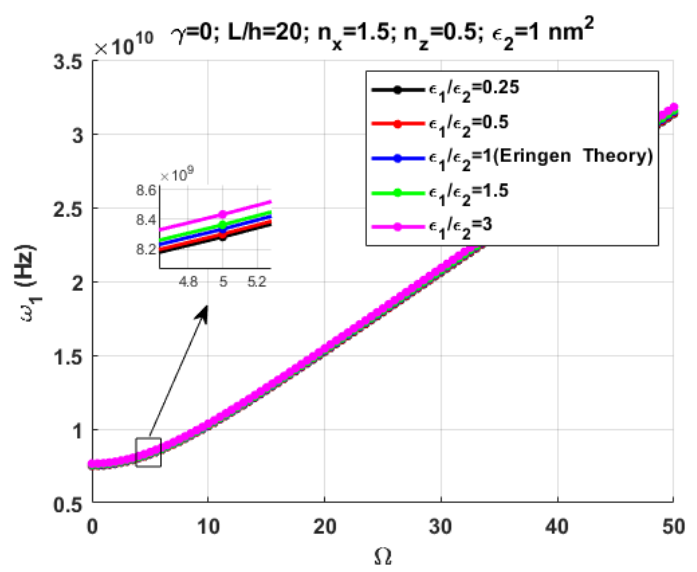

(d)

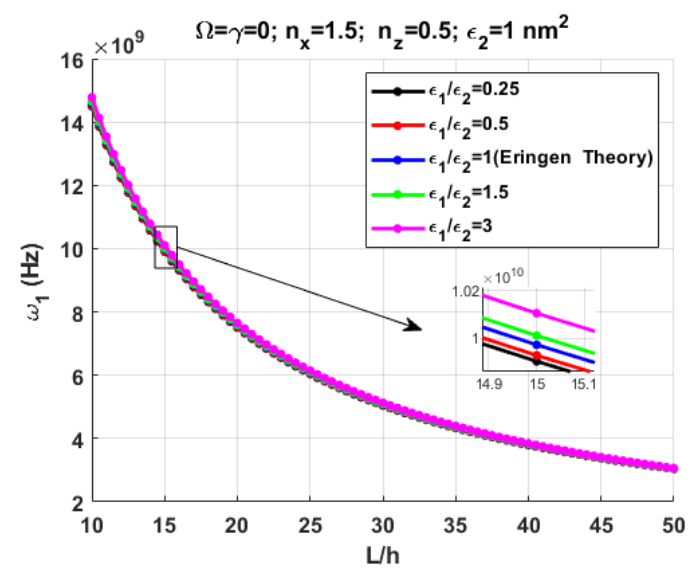

(e)

Fig 10. The effects of nonlocal parameters $\left(\epsilon_{1}, \epsilon_{2}\right)$ on the fundamental natural frequency for
varying (a) $n x$
(b) $n z$
(c) $\gamma$
(d) $\Omega$
(e) $L / h$ 
To show the effect of the nonlocal parameters we assume that $\epsilon_{2}=1 \mathrm{~nm}^{2}$ and is constant along the length of the beam. Then, by altering the ratio of $\epsilon_{1}$ to $\epsilon_{2}$ from 0.25 to 3 in five separate steps, different values for $\epsilon_{1}$ are obtained and consequently the natural frequencies were calculated. Figure 10 illustrates the effect of the nonlocal parameters on the fundamental natural frequency based on the variation of $n_{x}, n_{z}, \gamma, \Omega$ and $L / h$. Tthe fundamental natural frequency decreases as the ratio of $\epsilon_{1}$ to $\epsilon_{2}$ decreases while $\epsilon_{2}$ is constant. For $\epsilon_{1}=\epsilon_{2}$ the GNT reduces to conventional ENT. Hence, employing conventional ENT to materials with different $\epsilon_{1}$ and $\epsilon_{2}$ cannot properly estimate the vibrational behavior of these materials and this is the main advantage of GNT which is employed in current work.

In previous works, the Eringen nonlocal parameter was assumed to be constant and did not change when the mechanical properties change. However, in this paper for the first time and for high accuracy, the constant nonlocal parameters $\epsilon_{1}$ and $\epsilon_{2}$ are considered to alter along longitudinal (x-axis) and thickness (z-axis) directions given by Eq. (36), in a similar way to the other mechanical properties. Figure 11 reports the comparison of the results which are extracted on the basis of the constant and varying values for $\epsilon_{1}$ and $\epsilon_{2}$. 


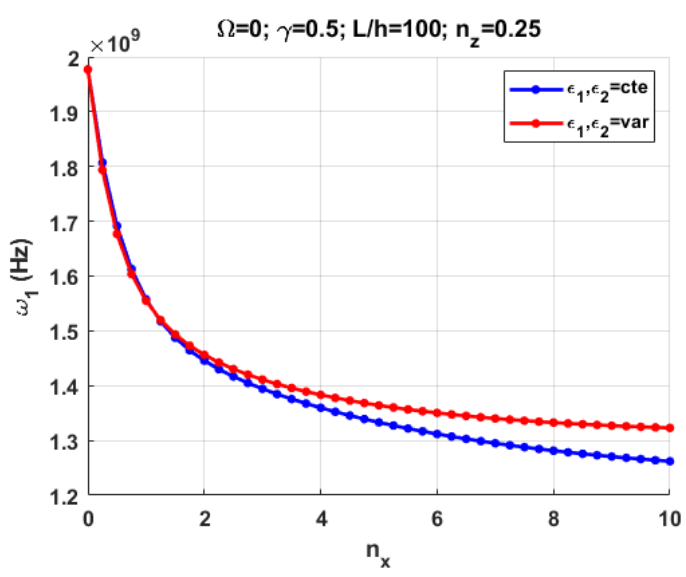

(a)

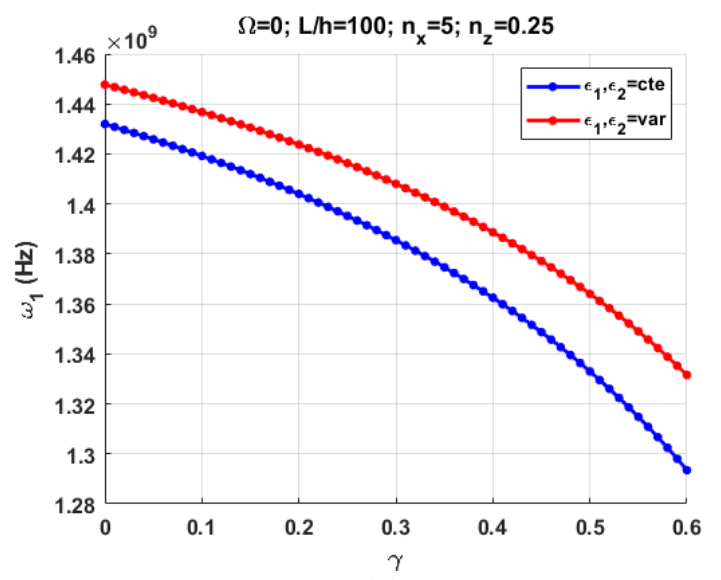

(c)

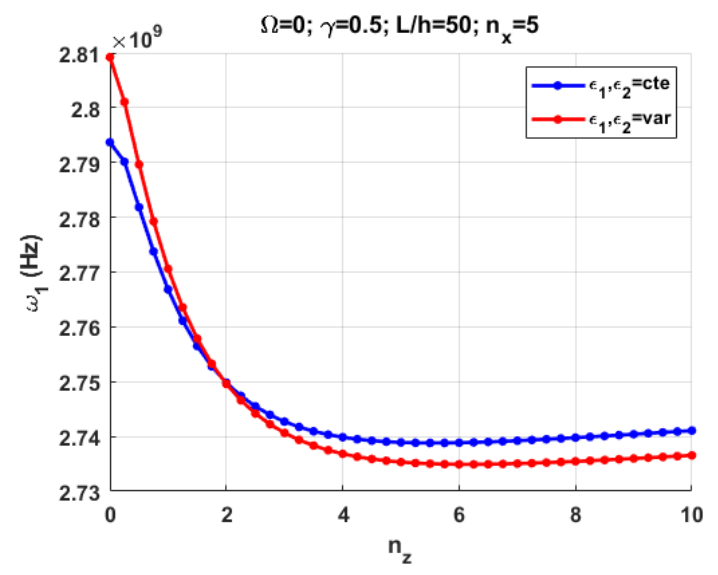

(b)

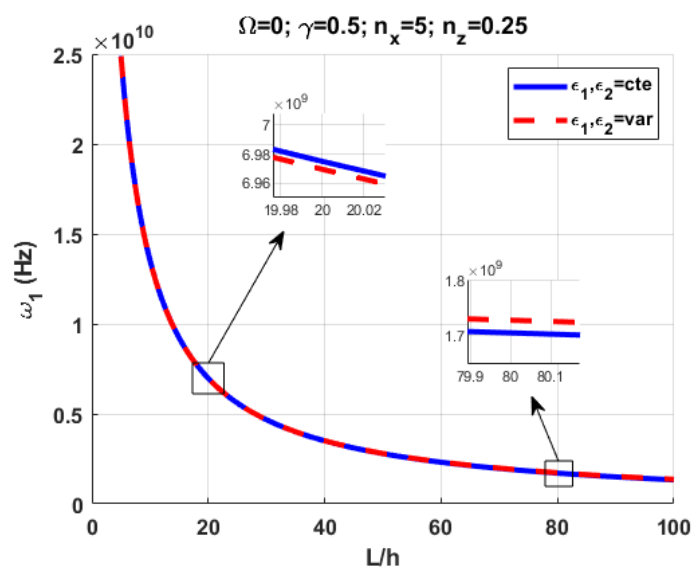

(d)

Fig 11. The effects of constant and varying nonlocal parameters $\left(\epsilon_{1}, \epsilon_{2}\right)$ on the fundamental natural $\begin{array}{lllll}\text { frequency with varying (a) } n x & \text { (b) } n z & \text { (c) } \gamma & \text { (d) } \Omega & \text { (e) } L / h\end{array}$

Thus, using constant values for the nonlocal parameters $\left(\epsilon_{1}, \epsilon_{2}\right)$ when the material properties change, leads to a considerable deviation in the results. Although, in Fig. 11(d), the deviation due to the nonlocal parameters is small in comparison to the variation due to $L / h$.

\subsection{The Effect of Boundary Conditions}

Figure 12 shows the fundamental natural frequency for clamped (CC), clamped-simply supported (CS) and simply supported (SS) boundary conditions with variations in $n_{x}, n_{z}, \gamma, \Omega$ and $L / h$. The general behavior of the SS and CS nano-beams is the same as for the CC boundary conditions. Since the clamped boundary condition has one fewer degree of freedom than a simply supported bounry condition, the effectis to stiffen the beam; thus the natural frequencies 
for the SS nano-beams are lower than those for the CS nano-beams, which in turn are lower than those for the CC nano-beams, as illustrated in Fig. 12.

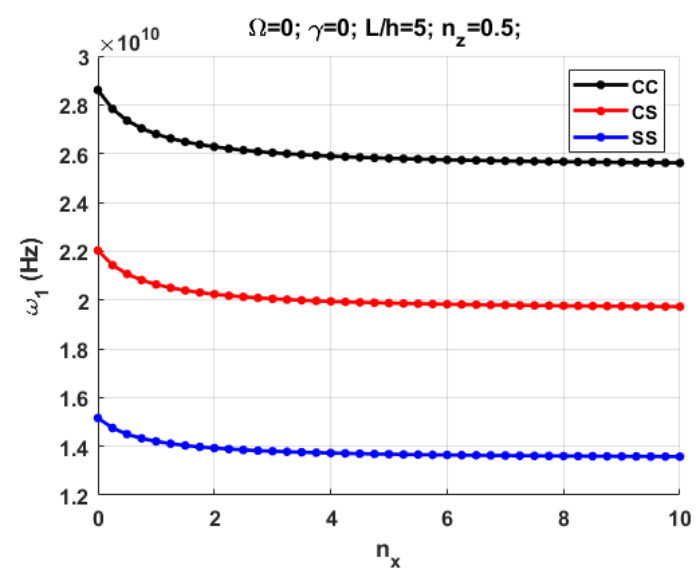

(a)

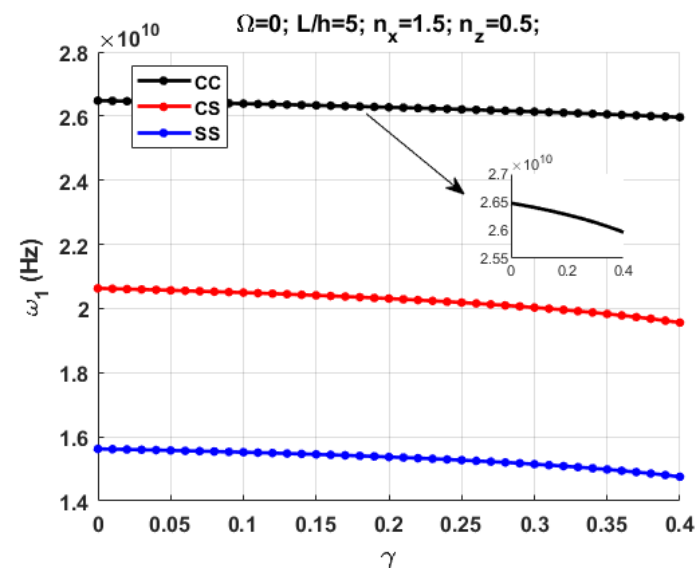

(c)

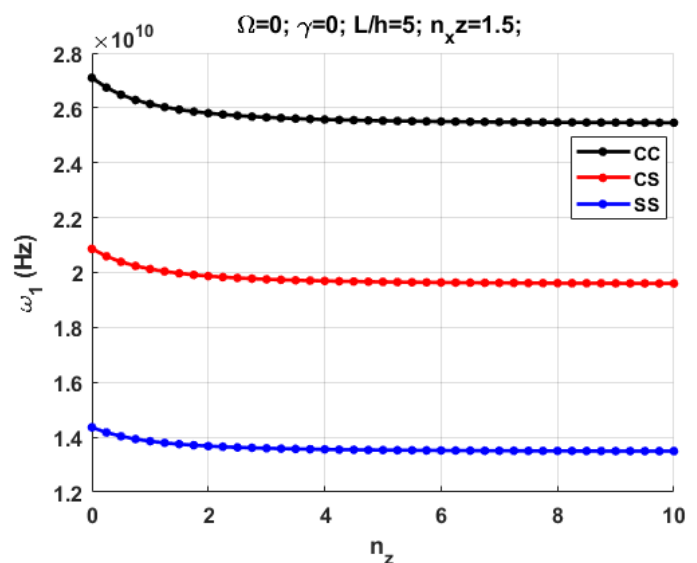

(b)

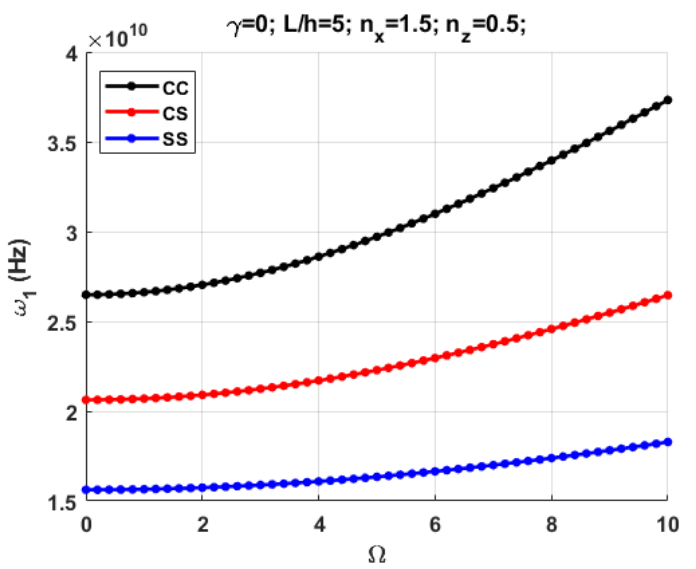

(d)

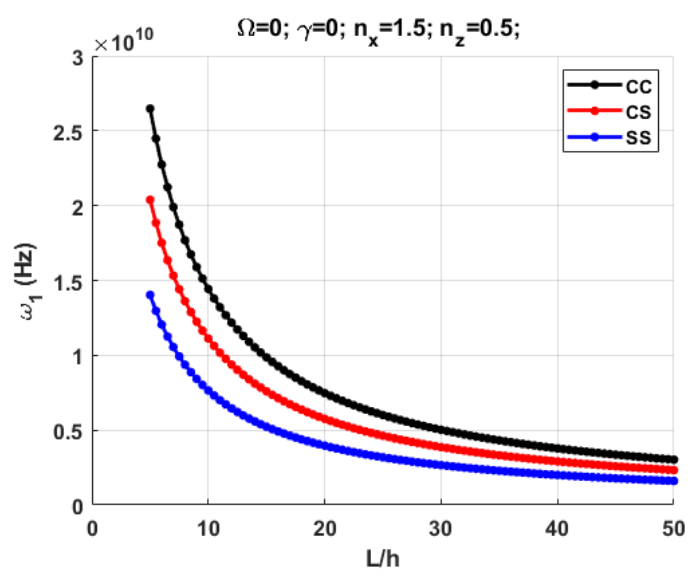

(e)

Fig 12. The fundamental natural frequency for different boundary conditions. (a) $n x$

(b)
$n z$
(c) $\gamma$
(d) $\Omega$
(e) $L / h$ 


\section{Conclusion}

The goal of this paper is to present a comprehensive mathematical model to investigate the vibrational behavior of a porous rotating $2 \mathrm{D}-\mathrm{FG}$ nanobeam, which is a configuration that is novel. The general nonlocal model is applied for the first time to derive the mathematical model of the rotating nanobeam. Furthermore, to establish a comprehensive study, a higher order shear deformation beam model is employed to develop the equations of motion. To solve the governing equations and obtain the results and calculate the natural frequencies, GDQM is utilized. The effects of material variation along the thickness and length directions, the rotating velocity of the nanobeams, the porosity volume fraction and the length to thickness ratio of the rotating nanobeams are calculated and discussed in detail. The main important results are summarized as follows:

1- Increasing $n z$ and $n x$ decreases the natural frequency. Also, for higher values of $n z$ and $n x$, the frequencies approach a constant value.

2- Increasing the nanobeam rotating velocity up to the corner velocity causes an increase in the fundamental natural frequency. However, increasing $\Omega$ over the corner rotating velocity $\Omega_{c}$ has no effect on the natural frequency and this is due to the $\mathrm{CC}$ boundary condition.

3- The value of the corner rotating velocity depends on the value of $n z$ and $n x$.

4- The variation of $\gamma$ has no significant effect on the corner rotating velocity.

5- The effects of $\gamma$ on the fundamental natural frequency depends on the values of $n z$ and $n x$. For different values of $n z$ and $n x$, the character of the variation of natural frequency with $\gamma$ can change.

6- Increasing $L / h$, not only decreases the natural frequency for $\Omega<\Omega_{c}$, but also increases the corner rotating velocity.

7- Increasing $L / h$ causes the natural frequency to decrease.

8- The fundamental natural frequencies decrease with a decrease in the ratio of $\epsilon_{1}$ to $\epsilon_{2}$ when $\epsilon_{1}$ is constant.

9- For materials with different $\epsilon_{1}$ and $\epsilon_{2}$, employing the conventional ENT cannot properly estimate the vibrational behavior. 
10- For all situations, the non-dimensional frequencies of SS nano-beams are lower than those of CS nano-beams, which are lower than those of CC nano-beams.

\section{References:}

[1] A. C. Eringen, Nonlocal continuum field theories. Springer Science \& Business Media, 2002.

[2] F. Yang, A. Chong, D. C. C. Lam, and P. Tong, "Couple stress based strain gradient theory for elasticity," International Journal of Solids and Structures, vol. 39, no. 10, pp. 2731-2743, 2002.

[3] D. C. Lam, F. Yang, A. Chong, J. Wang, and P. Tong, "Experiments and theory in strain gradient elasticity," Journal of the Mechanics and Physics of Solids, vol. 51, no. 8, pp. 1477-1508, 2003.

[4] M. Shaat and A. Abdelkefi, "New insights on the applicability of Eringen's nonlocal theory," International Journal of Mechanical Sciences, vol. 121, pp. 67-75, 2017.

[5] M. Ghadiri, S. Hosseini, and N. Shafiei, "A power series for vibration of a rotating nanobeam with considering thermal effect," Mechanics of Advanced Materials and Structures, vol. 23, no. 12, pp. 1414-1420, 2016.

[6] N. Shafiei, M. Kazemi, and L. Fatahi, "Transverse vibration of rotary tapered microbeam based on modified couple stress theory and generalized differential quadrature element method," Mechanics of Advanced Materials and Structures, vol. 24, no. 3, pp. 240-252, 2017.

[7] M. Mahinzare, H. Ranjbarpur, and M. Ghadiri, "Free vibration analysis of a rotary smart two directional functionally graded piezoelectric material in axial symmetry circular nanoplate," Mechanical Systems and Signal Processing, vol. 100, pp. 188-207, 2018.

[8] M. Azimi, S. S. Mirjavadi, N. Shafiei, A. Hamouda, and E. Davari, "Vibration of rotating functionally graded Timoshenko nano-beams with nonlinear thermal distribution," Mechanics of Advanced Materials and Structures, vol. 25, no. 6, pp. 467-480, 2018.

[9] M. Mahinzare, M. J. Alipour, S. A. Sadatsakkak, and M. Ghadiri, "A nonlocal strain gradient theory for dynamic modeling of a rotary thermo piezo electrically actuated nano FG circular plate," Mechanical Systems and Signal Processing, vol. 115, pp. 323-337, 2019.

[10] C. Roque, A. Ferreira, and J. Reddy, "Analysis of Timoshenko nanobeams with a nonlocal formulation and meshless method," International journal of engineering science, vol. 49, no. 9, pp. 976-984, 2011.

[11] R. Ansari, R. Gholami, and S. Sahmani, "Free vibration analysis of size-dependent functionally graded microbeams based on the strain gradient Timoshenko beam theory," Composite Structures, vol. 94, no. 1, pp. 221-228, 2011.

[12] H.-T. Thai, "A nonlocal beam theory for bending, buckling, and vibration of nanobeams," International journal of engineering science, vol. 52, pp. 56-64, 2012. 
[13] J. Aranda-Ruiz, J. Loya, and J. Fernández-Sáez, "Bending vibrations of rotating nonuniform nanocantilevers using the Eringen nonlocal elasticity theory," Composite Structures, vol. 94, no. 9, pp. 2990-3001, 2012.

[14] R. Sourki and S. Hosseini, "Coupling effects of nonlocal and modified couple stress theories incorporating surface energy on analytical transverse vibration of a weakened nanobeam," The European Physical Journal Plus, vol. 132, no. 4, p. 184, 2017.

[15] A. Babaei and A. Rahmani, "On dynamic-vibration analysis of temperature-dependent Timoshenko microbeam possessing mutable nonclassical length scale parameter," Mechanics of Advanced Materials and Structures, pp. 1-8, 2018.

[16] H. B. Khaniki, "On vibrations of nanobeam systems," International journal of engineering science, vol. 124, pp. 85-103, 2018.

[17] H. B. Khaniki, "Vibration analysis of rotating nanobeam systems using Eringen's twophase local/nonlocal model," Physica E: Low-dimensional Systems and Nanostructures, vol. 99, pp. 310-319, 2018.

[18] F. Ebrahimi and M. R. Barati, "Vibration analysis of nonlocal beams made of functionally graded material in thermal environment," The European Physical Journal Plus, vol. 131, no. 8, p. 279, 2016.

[19] F. Ebrahimi and M. R. Barati, "Surface effects on the vibration behavior of flexoelectric nanobeams based on nonlocal elasticity theory," The European Physical Journal Plus, vol. 132, no. 1, p. 19, 2017.

[20] C. Roque, P. Martins, A. Ferreira, and R. Jorge, "Differential evolution for free vibration optimization of functionally graded nano beams," Composite Structures, vol. 156, pp. 2934, 2016.

[21] N. Shafiei and G.-L. She, "On vibration of functionally graded nano-tubes in the thermal environment," International journal of engineering science, vol. 133, pp. 84-98, 2018.

[22] S. Pradhan and T. Murmu, "Application of nonlocal elasticity and DQM in the flapwise bending vibration of a rotating nanocantilever," Physica E: Low-dimensional Systems and Nanostructures, vol. 42, no. 7, pp. 1944-1949, 2010.

[23] M. Mohammadi, M. Safarabadi, A. Rastgoo, and A. Farajpour, "Hygro-mechanical vibration analysis of a rotating viscoelastic nanobeam embedded in a visco-Pasternak elastic medium and in a nonlinear thermal environment," Acta Mechanica, vol. 227, no. 8, pp. 2207-2232, 2016.

[24] F. Ebrahimi and N. Shafiei, "Application of Eringens nonlocal elasticity theory for vibration analysis of rotating functionally graded nanobeams," Smart Structures and Systems, vol. 17, no. 5, pp. 837-857, 2016.

[25] N. Shafiei, S. S. Mirjavadi, B. MohaselAfshari, S. Rabby, and M. Kazemi, "Vibration of two-dimensional imperfect functionally graded (2D-FG) porous nano-/micro-beams," Computer Methods in Applied Mechanics and Engineering, vol. 322, pp. 615-632, 2017.

[26] Y. Tang and T. Yang, "Bi-directional functionally graded nanotubes: fluid conveying dynamics," International Journal of Applied Mechanics, vol. 10, no. 04, p. 1850041, 2018. 
[27] Y. Tang and T. Yang, "Post-buckling behavior and nonlinear vibration analysis of a fluid-conveying pipe composed of functionally graded material," Composite Structures, vol. 185, pp. 393-400, 2018.

[28] Y. Tang and Q. Ding, "Nonlinear vibration analysis of a bi-directional functionally graded beam under hygro-thermal loads," Composite Structures, vol. 225, p. 111076, 2019.

[29] Y. Tang, X. Lv, and T. Yang, "Bi-directional functionally graded beams: asymmetric modes and nonlinear free vibration," Composites Part B: Engineering, vol. 156, pp. 319$331,2019$.

[30] S. S. Mirjavadi, B. Mohasel Afshari, N. Shafiei, S. Rabby, and M. Kazemi, "Effect of temperature and porosity on the vibration behavior of two-dimensional functionally graded micro-scale Timoshenko beam," Journal of Vibration and Control, vol. 24, no. 18, pp. 4211-4225, 2018.

[31] F. Ebrahimi and M. R. Barati, "A modified nonlocal couple stress-based beam model for vibration analysis of higher-order FG nanobeams," Mechanics of Advanced Materials and Structures, vol. 25, no. 13, pp. 1121-1132, 2018.

[32] N. Shafiei, M. Ghadiri, and M. Mahinzare, "Flapwise bending vibration analysis of rotary tapered functionally graded nanobeam in thermal environment," Mechanics of Advanced Materials and Structures, vol. 26, no. 2, pp. 139-155, 2019.

[33] J. Fang, D. Zhou, and Y. Dong, "Three-dimensional vibration of rotating functionally graded beams," Journal of Vibration and Control, vol. 24, no. 15, pp. 3292-3306, 2018.

[34] J. Fang, J. Gu, and H. Wang, "Size-dependent three-dimensional free vibration of rotating functionally graded microbeams based on a modified couple stress theory," International Journal of Mechanical Sciences, vol. 136, pp. 188-199, 2018.

[35] R. Talebitooti, S. O. Rezazadeh, and A. Amiri, "Comprehensive semi-analytical vibration analysis of rotating tapered AFG nanobeams based on nonlocal elasticity theory considering various boundary conditions via differential transformation method," Composites Part B: Engineering, vol. 160, pp. 412-435, 2019.

[36] S. Bhattacharya and D. Das, "Free vibration analysis of bidirectional-functionally graded and double-tapered rotating micro-beam in thermal environment using modified couple stress theory," Composite Structures, vol. 215, pp. 471-492, 2019.

[37] A. Aria and M. Friswell, "A nonlocal finite element model for buckling and vibration of functionally graded nanobeams," Composites Part B: Engineering, vol. 166, pp. 233-246, 2019.

[38] H. B. Khaniki, "On vibrations of FG nanobeams," International journal of engineering science, vol. 135, pp. 23-36, 2019.

[39] M. S. Atanasov and V. Stojanović, "Nonlocal forced vibrations of rotating cantilever nano-beams," European Journal of Mechanics-A/Solids, vol. 79, p. 103850, 2020.

[40] K. Kiani and S. Soltani, "Nonlocal longitudinal, flapwise, and chordwise vibrations of rotary doubly coaxial/non-coaxial nanobeams as nanomotors," International Journal of Mechanical Sciences, vol. 168, p. 105291, 2020. 
[41] T. Yang, Y. Tang, Q. Li, and X.-D. Yang, "Nonlinear bending, buckling and vibration of bi-directional functionally graded nanobeams," Composite Structures, vol. 204, pp. 313319, 2018.

[42] M. Shaat, "A general nonlocal theory and its approximations for slowly varying acoustic waves," International Journal of Mechanical Sciences, vol. 130, pp. 52-63, 2017.

[43] S. Faroughi and M. Shaat, "Poisson's ratio effects on the mechanics of auxetic nanobeams," European Journal of Mechanics-A/Solids, vol. 70, pp. 8-14, 2018.

[44] M. Shaat, "Correction of local elasticity for nonlocal residuals: application to EulerBernoulli beams," Meccanica, vol. 53, no. 11-12, pp. 3015-3035, 2018.

[45] X. Wang, "Differential Quadrature and Differential Quadrature Based Element Methods," ed: Elsevier, 2015.

[46] C. Lim, G. Zhang, and J. Reddy, "A higher-order nonlocal elasticity and strain gradient theory and its applications in wave propagation," Journal of the Mechanics and Physics of Solids, vol. 78, pp. 298-313, 2015.

[47] F. Ebrahimi and M. R. Barati, "A nonlocal higher-order shear deformation beam theory for vibration analysis of size-dependent functionally graded nanobeams," Arabian Journal for Science and Engineering, vol. 41, no. 5, pp. 1679-1690, 2016.

[48] P. Khodabakhshi and J. Reddy, "A unified beam theory with strain gradient effect and the von Kármán nonlinearity," ZAMM-Journal of Applied Mathematics and Mechanics/Zeitschrift für Angewandte Mathematik und Mechanik, vol. 97, no. 1, pp. 7091, 2017.

[49] A. Masoumi, A. Amiri, and R. Talebitooti, "Flexoelectric effects on wave propagation responses of piezoelectric nanobeams via nonlocal strain gradient higher order beam model," Materials Research Express, vol. 6, no. 10, p. 1050d5, 2019. 


\section{Author Credit Statement}

Re: Revised revision MSSP20-49.

Manuscript title: The Vibration of Two-Dimensional Imperfect Functionally Graded (2D-FG)

Porous Rotating Nanobeams based on General Nonlocal Theory

Authors: Arash Rahmani ${ }^{\mathrm{a}}$, Shirko Faroughi ${ }^{\mathrm{a}}$, Michael I. Friswell ${ }^{\mathrm{b}}$

Arash Rahmani: Methodology, Software, Writing - Original Draft

Shirko Faroughi: Conceptualization, Methodology, Writing - Original Draft, Supervision

Michael I. Friswell: Writing - Review \& Editing, Supervision 